\title{
1 Thermal stability criterion of complex reactions for batch processes
}

2

5 Abstract

6 Thermal stability of batch processes is a major factor for the safe and efficient production 7 of polymers and pharmaceutical chemicals. The prediction of the thermal stability for such 8 processes was shown in Kähm and Vassiliadis (2018d) to be unreliable with most stability 9 criteria found in literature also presenting a novel criterion, $\mathcal{K}$, which was shown to give

Walter Kähm*, Vassilios S. Vassiliadis

Cambridge, West Cambridge Site, Philippa Fawcett Drive, CB3 0AS Cambridge, UK reliable stability predictions for single reactions of higher order.

This work provides a detailed derivation for the generalization of thermal stability criterion $\mathcal{K}$ applied to reaction networks of arbitrary complexity, consisting of parallel and competing reactions of both exothermic and endothermic nature. The generalized thermal stability criterion $\mathcal{K}$ is then applied to Model Predictive Control (MPC) frameworks to intensify batch processes in a safe manner, reducing the time required to reach the target conversion. Several illustrative computational case studies are presented, highlighting the proposed methodology and verifying its validity.

Keywords: thermal stability criterion, batch process, process control, process intensification

${ }^{*}$ Corresponding author

Email address: wk263@cam.ac.uk (Walter Kähm) 


\begin{tabular}{|c|c|c|}
\hline \multicolumn{3}{|c|}{$\begin{array}{ll}19 & \text { Nomenclature } \\
20 & \text { Roman Symbols }\end{array}$} \\
\hline 21 & CSTR & Continuous Stirred Tank Reactor \\
\hline 22 & MPC & Model Predictive Control \\
\hline 23 & $\mathrm{OCP}$ & Optimal Control Problem \\
\hline 24 & $\mathrm{PI} / \mathrm{PID}$ & Proportional-Integral / Proportional-Integral-Differential control \\
\hline $\begin{array}{l}25 \\
26\end{array}$ & $\mathrm{TT}, \mathrm{TIC}$ & $\begin{array}{l}\text { temperature transmitter and temperature integrated controller, re- } \\
\text { spectively }\end{array}$ \\
\hline & & \\
\hline & $A$ & heat transfer area between reactor contents and cooling jacket $\left[\mathrm{m}^{2}\right]$ \\
\hline 29 & $B$ & Barkelew number $[-]$ \\
\hline 30 & $\mathrm{~A}, \mathrm{~B}, \mathrm{C}, \mathrm{D}, \mathrm{E}, \mathrm{F}, \mathrm{G}$ & components for each reaction $[-]$ \\
\hline 31 & {$[\mathrm{~A}]$} & concentration of component $\mathrm{A}\left[\mathrm{kmol} \mathrm{m}^{-3}\right]$ \\
\hline 32 & $C_{p}$ & heat capacity $\left[\mathrm{kJ} \mathrm{mol}^{-1} \mathrm{~K}^{-1}\right]$ \\
\hline 33 & $\mathrm{Da}, \mathrm{Da}_{\mathrm{res}}$ & Damköhler number and resultant Damköhler number, respectively [-] \\
\hline 34 & $\Delta H_{r}$ & heat of reaction $\left[\mathrm{J} \mathrm{kmol}^{-1}\right]$ \\
\hline 35 & $E_{a}$ & activation energy $\left[\mathrm{J} \mathrm{kmol}^{-1}\right]$ \\
\hline 36 & $f$ & generic function $[-]$ \\
\hline 37 & $g$ & differential equation $[-]$ \\
\hline 38 & $h$ & algebraic equation $[-]$ \\
\hline 39 & $\mathbf{J}$ & Jacobian matrix $[-]$ \\
\hline 40 & $k_{0}$ & pre-exponential Arrhenius factor $[-]$ \\
\hline 41 & $K_{P}$ & proportional constant for PI controller $\left[\mathrm{m}^{3} \mathrm{~K}^{-1} \mathrm{~s}^{-1}\right]$ \\
\hline 42 & $M$ & number of reactions within a reaction network $[-]$ \\
\hline 43 & $m_{B}, m_{\text {Dares }}, m_{\gamma}, m_{\mathrm{St}}$ & gradient coefficients with respect to $B, \mathrm{Da}_{\mathrm{res}}, \gamma$ and St $[-]$ \\
\hline 44 & $N$ & number of reagents within a reaction network $[-]$ \\
\hline 45 & $n$ & reaction order $[-]$ \\
\hline 46 & $q$ & volumetric flow rate $\left[\mathrm{m}^{3} \mathrm{~s}^{-1}\right]$ \\
\hline 47 & $R$ & universal molar gas constant $\left[\mathrm{J} \mathrm{kmol}^{-1} \mathrm{~K}^{-1}\right]$ \\
\hline 18 & $r$ & reaction rate $\left[\mathrm{kmol} \mathrm{m}^{-3} \mathrm{~s}^{-1}\right]$ \\
\hline
\end{tabular}




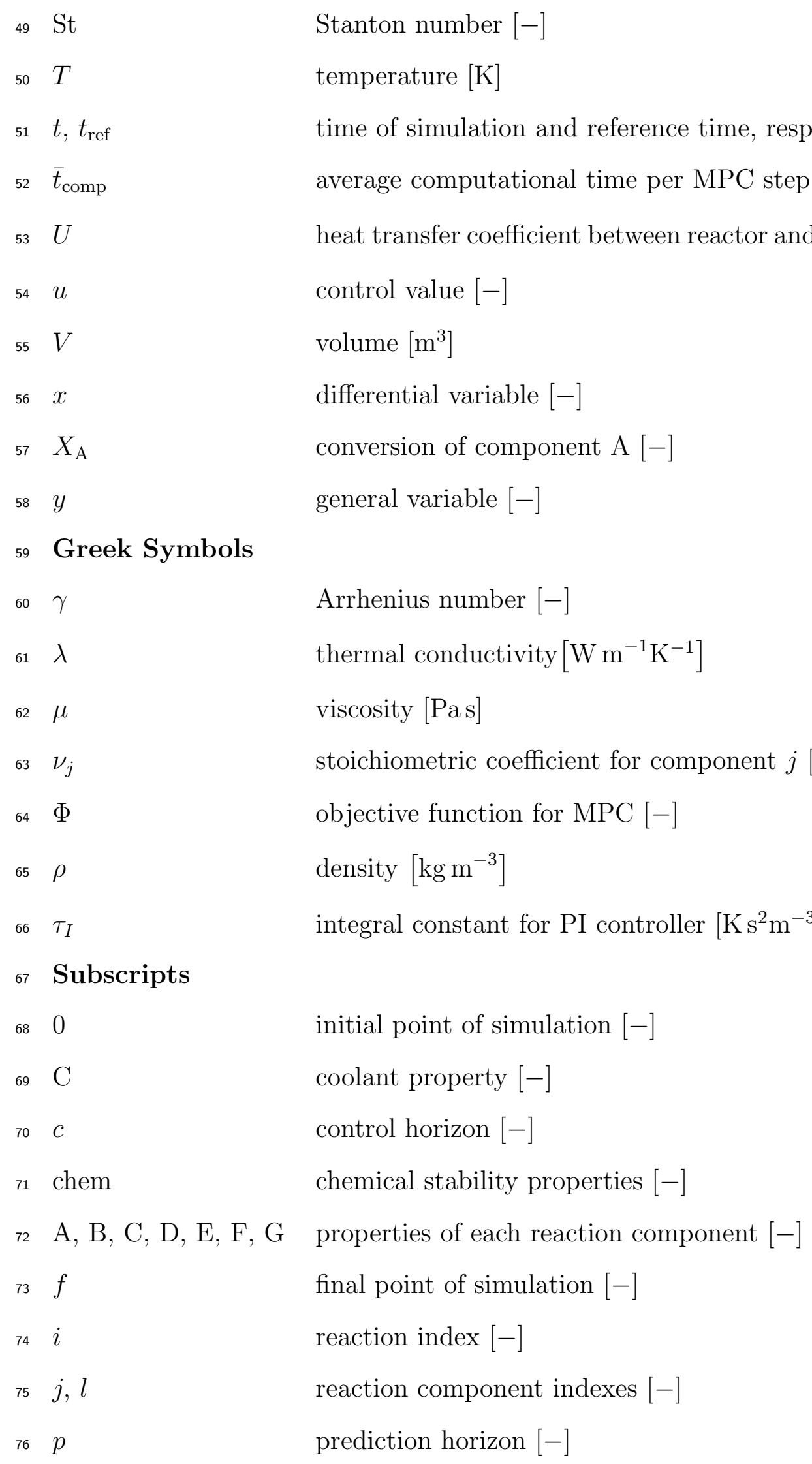


77 peak

${ }_{78} \mathrm{R}$

79 reac

$80 \quad \mathrm{sp}$

81

82

83

$\mathcal{D}$

properties at the peak temperature during the process [-]

reacting mixture property $[-]$

properties at the end of the reaction $[-]$

set-point $[-]$

time step for simulations $[-]$

\section{Other Symbols}

contribution to the divergence of the Jacobian due to single reaction $\left[\mathrm{S}^{-1}\right]$

estimate of the divergence at boundary of instability $\left[\mathrm{s}^{-1}\right]$

thermal stability criterion $\left[\mathrm{s}^{-1}\right]$

\section{Introduction}

Exothermic chemical reactions carried out in batch reactors are an essential part for process control in industry. Of great importance is the adjustment of the set-point temperature in order to ensure safe operation of reactors. The loss of thermal stability in exothermic reactions leads to an uncontrolled increase of temperature, having detrimental effects in terms of the ecology and the economics of industrial plants (Theis, 2014). This effect is due to the potentially large increase in pressure, causing the release of hazardous chemicals, as well as an unsafe environment for workers.

For this reason a method to determine the thermal stability of batch processes is required. The chemical stability of reagents, products and materials sets an upper limit to the reaction temperature which must not be exceeded to avoid by-product formation and safety issues.

The thermal stability has to consider the dynamic behavior of the system including temperature and concentration profiles, reaction kinetics and heat transfer to the cooling jacket. In many batch processes thermal stability is the limiting factor for more efficient operation.

Most control systems for batch reactors make use of Proportional-Integral-Differential (PID) controllers, setting a constant set-point temperature throughout the process (Winde, 2009). As the reaction proceeds, the cooling required reduces as the amount of reagents present usually decreases over time, therefore reducing the heat generation.

Model Predictive Control (MPC) enables to include such a stability constraint within a more flexible control scheme, which can further be implemented in industry. MPC continuously updates the reaction temperature set-point whilst taking into account system constraints (Chuong La et al., 2017), which PID control cannot (Winde, 2009). A fundamental 
requirement for the application of MPC to industrial systems is the reliable and quick detection of stability during the process.

Stability criteria found in literature work well for continuous stirred tank reactors (CSTRs), e.g. the theory of heat explosion (Semenov, 1940), Lyapunov functions (Huang et al., 2012), the Routh-Hurwitz criterion (Anagnost and Desoer, 1991). These methods are found not to work well to predict the thermal stability for batch reactor systems.

In Rossi et al. (2015) a boolean function is defined to identify the stability of fed-batch reactor systems which is included as a barrier function (Nocedal and Wright, 2006). Problems arise with this method, which are outlined in Kähm and Vassiliadis (2018c).

Stability criteria based on Lyapunov functions were implemented in systems operating at steady state before, for which a good review is given by Albalawi et al. (2018). For continuous systems in industry good results were obtained with such an approach (Zhang et al., 2018). This work cannot be easily transferred to batch reactors, which is why further investigation is required.

The structure of embedding stability criteria as additional system constraints within an MPC framework are present in literature (Zhang et al., 2018). These systems are limited to continuous systems, for which a steady-state operating point exists. This work tackles the same issue, but for batch processes which are inherently non-steady state.

The divergence method (Strozzi and Zaldívar, 1999), as was shown in Kähm and Vassiliadis (2018d,c), results in stability predictions which are systematically too conservative for batch processes. This makes it unusable for process intensification.

The Lyapunov exponent method (Strozzi and Zaldívar, 1994) results in reliable prediction of system stability after tuning of the initial perturbation and time frame used (Kähm and Vassiliadis, 2018a,b). The analysis of the computational time showed that for large reaction systems this method might reach limits of applicability for industrial scale problems.

The unreliable nature of the divergence method and the potentially large computational time to evaluate Lyapunov exponents hence requires the development of an alternative stability criterion. Thermal stability criterion $\mathcal{K}$ predicts the stability of batch processes reliably, as well as results in short computational times when embedded within an MPC framework (Kähm and Vassiliadis, 2018d,c). This work is focused on achieving the following goals:

- Extension of stability criterion $\mathcal{K}$ for complex reaction networks

- Validation of criterion $\mathcal{K}$ by comparison with unstable reaction profiles

- Intensification of batch processes by MPC with embedded stability analysis based on criterion $\mathcal{K}$ 
- Analysis of the computational time for the MPC frameworks with and without embedded stability constraints

Achieving the above goals enables the successful implementation of stability criterion $\mathcal{K}$ for industrial systems, in which reaction networks of considerable size are present.

This paper is organized as follows: in Section 2 stability criterion $\mathcal{K}$ together with the underlying model structure is introduced, and the extension to several simultaneous reactions is outlined. Section 3 contains the batch reactor model with the chemical reaction schemes analyzed in this work. The validity of the extension of stability criterion $\mathcal{K}$ is tested in Section 4. In Section 5 the newly developed form of stability criterion $\mathcal{K}$ is applied to batch processes together with MPC to intensify these processes. Section 6 finishes this work by summarizing the key results and outlining future work necessary for the successful implementation of this control scheme.

\section{Stability criterion $\mathcal{K}$}

\subsection{Properties and description of stability criterion $\mathcal{K}$}

Stability criterion $\mathcal{K}$ describes the transition of thermal instability in batch reactors. For a thermally stable process, the criterion should give a value of:

$$
\mathcal{K} \leq 0
$$

An unstable reaction is obtained when the value of the criterion becomes positive:

$$
\mathcal{K}>0
$$

The stability criterion $\mathcal{K}$ is based on the difference between the divergence of the Jacobian of the relevant system variables and the correction function $\mathcal{E}$ (Kähm and Vassiliadis, 2018d,c). At each current time step $(s)$ stability criterion $\mathcal{K}^{(s)}$ is given by:

$$
\mathcal{K}^{(s)}=\operatorname{div}\left[\mathbf{J}^{(s)}\right]-\left|\mathcal{E}^{(s)}\right|
$$

The correction function $\mathcal{E}^{(s)}$ was derived in Kähm and Vassiliadis (2018d) as a function of the divergence of the Jacobian at the previous time step $(s-1) \operatorname{div}\left[\mathbf{J}^{(s-1)}\right]$, and the following dimensionless numbers: Damköhler number Da, Barkelew number $B$, Arrhenius number $\gamma$, and the Stanton number St. The function for $\mathcal{E}^{(s)}$ represents the linear estimate of the divergence $\operatorname{div}\left[\mathbf{J}^{(s)}\right]$ at the boundary of instability, dependent on the following variables:

$$
\mathcal{E}^{(s)}=f\left(\operatorname{div}\left[\mathbf{J}^{(s-1)}\right], B^{(s)}, B^{(s-1)}, \gamma^{(s)}, \gamma^{(s-1)}, \mathrm{Da}^{(s)}, \mathrm{Da}^{(s-1)}, \mathrm{St}^{(s)}, \mathrm{St}^{(s-1)}\right)
$$


From Equation (2.4) it can be seen that the value of the linear estimate at time step $(s), \mathcal{E}^{(s)}$, uses information from the current time step $(s)$ and the previous time step $(s-1)$. This function is sought after in order to correct for the fact that the value of the divergence $\operatorname{div}\left[\mathbf{J}^{(s)}\right]$ does not correctly predict when thermal runaway behavior occurs.

In Kähm and Vassiliadis (2018c) the thermal stability criterion $\mathcal{K}$ was derived for a reaction of the following form:

$$
\nu_{\mathrm{A}} \mathrm{A}+\nu_{\mathrm{B}} \mathrm{B} \rightarrow \mathrm{C}
$$

where $\nu_{\mathrm{A}}$ and $\nu_{\mathrm{B}}$ are stoichiometric coefficients for components $\mathrm{A}$ and $\mathrm{B}$. The rate of the reaction given in Equation (2.5) depends on both components according to the Arrhenius expression (Davis and Davis, 2003).

The relevant variables for a thermal runaway are the ones that contribute towards the heat generation in the reactor system (Bosch et al., 2004). In Kähm and Vassiliadis (2018c) it was shown that the divergence of the Jacobian for a batch reactor system with a reaction according to Equation (2.5) is given by:

$$
\begin{aligned}
\operatorname{div}[\mathbf{J}] t_{\text {ref }}= & -\left(\nu_{\mathrm{A}} n_{\mathrm{A}} \mathrm{Da}_{\mathrm{A}}+\nu_{\mathrm{B}} n_{\mathrm{B}} \mathrm{Da}_{\mathrm{B}}\right) \exp (-\gamma) \\
& +B \gamma \mathrm{Da}_{\mathrm{A}} \exp (-\gamma)-\mathrm{St}
\end{aligned}
$$

and

$$
\begin{aligned}
B & =\frac{[\mathrm{A}]\left(-\Delta H_{r}\right)}{\rho_{\mathrm{R}} C_{p, \mathrm{R}} T_{\mathrm{R}}} \\
\gamma & =\frac{E_{a}}{R T_{\mathrm{R}}} \\
\mathrm{Da}_{\mathrm{A}} & =k_{0}[\mathrm{~A}]^{n_{\mathrm{A}}-1}[\mathrm{~B}]^{n_{\mathrm{B}}} t_{\text {ref }} \\
\mathrm{Da}_{\mathrm{B}} & =k_{0}[\mathrm{~A}]^{n_{\mathrm{A}}}[\mathrm{B}]^{n_{\mathrm{B}}-1} t_{\text {ref }} \\
\mathrm{St} & =\frac{U A}{\rho_{\mathrm{R}} C_{p, \mathrm{R}} V_{\mathrm{R}}} t_{\mathrm{ref}}
\end{aligned}
$$

where $B$ is the Barkelew number, $\gamma$ is the Arrhenius number, $\mathrm{Da}_{\mathrm{A}}$ and $\mathrm{Da}$ a are the Damköhler numbers for components A and B, respectively, and St is the Stanton number. The reference time $t_{\text {ref }}$ is necessary as the units of the divergence are given by $\left[\mathrm{s}^{-1}\right]$. Therefore the introduction of $t_{\text {ref }}$ ensures each variable in Equation (2.7) is dimensionless. In the further derivation this variable will cancel out. The other variables within Equation (2.7) are the Arrhenius pre-exponential factor $k_{0}$, the concentrations of components $\mathrm{A}$ and $\mathrm{B}$ given by $[\mathrm{A}]$ and $[\mathrm{B}]$, respectively, the reaction orders $n_{\mathrm{A}}$ and $n_{\mathrm{B}}$, the activation energy $E_{a}$, the universal molar 
gas constant $R$, the reactor temperature $T_{\mathrm{R}}$, the density of the reaction mixture $\rho_{\mathrm{R}}$, the heat capacity of the reaction mixture $C_{p, \mathrm{R}}$, the reactor volume $V_{\mathrm{R}}$, the enthalpy of reaction $\Delta H_{r}$, the heat transfer coefficient $U$, and the heat transfer area of the cooling jacket $A$.

In Equation (2.7) it can be seen that two Damköhler numbers are present for each reagent in the reaction, each having the same Arrhenius factor of $k_{0}$. Hence in Kähm and Vassiliadis (2018c) it was shown that a resultant Damköhler number Da $_{\text {res }}$ can be introduced to simplify the derivation of criterion $\mathcal{K}$ by summarizing the effect of the single reaction:

$$
\mathrm{Da}_{\mathrm{res}}=\nu_{\mathrm{A}} n_{\mathrm{A}} \mathrm{Da}_{\mathrm{A}}+\nu_{\mathrm{B}} n_{\mathrm{B}} \mathrm{Da}_{\mathrm{B}}
$$

The simplification in Equation (2.8) allows for the derivation of criterion $\mathcal{K}$. From Equation (2.4) it is required to find $\mathcal{E}^{(s)}$ as a function of $\operatorname{div}\left[\mathbf{J}^{(s-1)}\right]$ and all dimensionless variables at time steps $(s-1)$ and $(s)$. The derivation of function $\mathcal{E}$ is based on the analysis carried out in Kähm and Vassiliadis (2018d). In this derivation it is shown that $\mathcal{E}^{(s)}$ is related to the divergence in steps $(s-1)$ and $(s)$ in the following manner:

$$
\mathcal{E}^{(s)}=\operatorname{div}\left[\mathbf{J}^{(s-1)}\right]+\operatorname{div}\left[\mathbf{J}^{(s-1)}\right] \cdot \operatorname{d} \ln \left(\operatorname{div}\left[\mathbf{J}^{(s)}\right]\right)
$$

The definition of function $\mathcal{E}^{(s)}$ states that it estimates the divergence of the Jacobian in time step $(s)$ if the system were to be at the boundary of instability. In Equations (4.4) and (4.5) in the work of Kähm and Vassiliadis (2018c) an expression for $d \ln \left(\operatorname{div}\left[\mathbf{J}^{(s)}\right]\right)$ is found, which results in the following expression for $\mathcal{E}^{(s)}$ :

$$
\begin{aligned}
\mathcal{E}^{(s)}= & \operatorname{div}\left[\mathbf{J}^{(s-1)}\right]+\operatorname{div}\left[\mathbf{J}^{(s-1)}\right] \cdot\left(m_{B} \frac{B^{(s)}-B^{(s-1)}}{B^{(s-1)}}+m_{\mathrm{Da}_{\mathrm{res}}} \frac{\mathrm{Da}_{\mathrm{res}}^{(s)}-\mathrm{Da}_{\mathrm{res}}^{(s-1)}}{\operatorname{Da}_{\mathrm{res}}^{(s-1)}}\right. \\
& \left.+m_{\gamma} \frac{\gamma^{(s)}-\gamma^{(s-1)}}{\gamma^{(s-1)}}+m_{\mathrm{St}} \frac{\mathrm{St}^{(s)}-\mathrm{St}^{(s-1)}}{\mathrm{St}^{(s-1)}}\right)
\end{aligned}
$$

where $m_{B}, m_{\mathrm{Da}_{\text {res }}}, m_{\gamma}$, and $m_{\mathrm{St}}$ are the gradient coefficients with respect to each dimensionless variables.

In Equation (2.10) it is shown that an expression for $\mathcal{E}^{(s)}$ is found using the variables shown in Equation (2.4). At time step $(s)$ the thermal stability criterion $\mathcal{K}^{(s)}$ is evaluated according to Equation (2.3) and the expression of $\mathcal{E}^{(s)}$ shown in Equation (2.10). The full expression for the single reaction given in Equation (2.5) is then: 


$$
\begin{aligned}
\mathcal{K}^{(s)}= & \operatorname{div}\left[\mathbf{J}^{(s)}\right]-\mid \operatorname{div}\left[\mathbf{J}^{(s-1)}\right]\left(1+m_{B} \frac{B^{(s)}-B^{(s-1)}}{B^{(s-1)}}+m_{\mathrm{Da}_{\mathrm{res}}} \frac{\mathrm{Da}_{\mathrm{res}}^{(s)}-\mathrm{Da}_{\mathrm{res}}^{(s-1)}}{\mathrm{Da}_{\mathrm{res}}^{(s-1)}}\right. \\
& \left.+m_{\gamma} \frac{\gamma^{(s)}-\gamma^{(s-1)}}{\gamma^{(s-1)}}+m_{\mathrm{St}} \frac{\mathrm{St}^{(s)}-\mathrm{St}^{(s-1)}}{\mathrm{St}^{(s-1)}}\right) \mid
\end{aligned}
$$

Similar expressions have to be derived for more complex reaction networks, consisting of several reactions. For this reason, the general mass and energy balances for such batch reactor systems are derived in the following section. Once a general expression for the divergence of the Jacobian is found, the generalization of thermal stability criterion $\mathcal{K}$ be formulated.

In Kähm and Vassiliadis (2018c) the gradient coefficients $m_{B}, m_{\gamma}, m_{\text {Dares }}$, and $m_{\mathrm{St}}$ were found for a reaction given by Equation (2.5). It was further shown that for large variations in reaction parameters such as the Arrhenius pre-exponential factor, enthalpy of reaction, activation energy, etc., constant values for the gradient coefficients are found. These values (Kähm and Vassiliadis, 2018c) are given by:

$$
\begin{aligned}
m_{B} & =1.28 \\
m_{\gamma} & =-21.8 \\
m_{\mathrm{Da}_{\mathrm{res}}} & =1.16 \\
m_{\mathrm{St}} & =-0.174
\end{aligned}
$$

The values given in Equation (2.12) are used for all simulations in this work, as they have been proven to work for complex single reactions in Kähm and Vassiliadis (2018c).

\subsection{Mass and energy balances for batch reactors}

The reaction rate of the reactions considered in this work are given by Arrhenius expressions (Davis and Davis, 2003). A single reaction rate for reaction $i$ within a network of $M$ reactions can be written as:

$$
r_{i}=k_{0, i} \exp \left(-\frac{E_{a, i}}{R T_{\mathrm{R}}}\right)[\mathrm{A}]^{n_{\mathrm{A}, i}}[\mathrm{~B}]^{n_{\mathrm{B}, i}} \quad i=1,2, \ldots, M
$$

where the constants in Equation (2.13) are the same as those given in Equation (2.7) related to a general reaction $i$. As a batch reactor is present, no in- or outflows are otherwise present, hence reducing the mass balances to reaction rates only. In the reaction considered in this example only components A and B are present. In general there can be any component with varying numbers. For clarity this form is used, which is generalized further in the following 
section.

For the batch reactors analyzed two sets of energy balances have to be considered: the reaction mixture and the cooling jacket. The energy balance of the reaction mixture, including the heat generated by the $M$ reactions, is given by the following expression:

$$
\frac{\mathrm{d}}{\mathrm{d} t}\left(\rho_{\mathrm{R}} C_{p, \mathrm{R}} T_{\mathrm{R}} V_{\mathrm{R}}\right)=\sum_{i=1}^{M}\left[r_{i}\left(\Delta H_{r, i}\right) V_{\mathrm{R}}\right]-U A\left(T_{\mathrm{R}}-T_{\mathrm{C}}\right)
$$

where $\Delta H_{r, i}$ is the enthalpy of reaction for reaction $i$, and $T_{\mathrm{C}}$ is the cooling jacket temperature.

The energy balances of the cooling jacket is given by the following expression:

$$
\frac{\mathrm{d}}{\mathrm{d} t}\left(\rho_{\mathrm{C}} C_{p, \mathrm{C}} T_{\mathrm{C}} V_{\mathrm{C}}\right)=q_{\mathrm{C}} \rho_{\mathrm{C}} C_{p, \mathrm{C}}\left(T_{\mathrm{C}, \text { in }}-T_{\mathrm{C}}\right)+U A\left(T_{\mathrm{R}}-T_{\mathrm{C}}\right)
$$

where $\rho_{\mathrm{C}}$ is the density of the coolant, $C_{p, \mathrm{C}}$ is the heat capacity of the coolant, $q_{\mathrm{C}}$ is the coolant flow through the cooling jacket, $V_{\mathrm{C}}$ is the cooling jacket volume, and $T_{\mathrm{C} \text {,in }}$ is the coolant inlet temperature. The heat produced by the stirrer within the reactor is negligible in comparison to the heat production by reaction and is therefore neglected in the further analysis.

\subsection{Generalization of criterion $\mathcal{K}$ for multiple reactions}

In this subsection the mass and energy balances for a total number of $M$ reactions with $N$ reagents are derived, which are then further used to find a generalized expression for the divergence of the Jacobian. This expression is then used to derive the generalized form of criterion $\mathcal{K}$.

\subsubsection{Divergence of Jacobian for general reaction systems}

The divergence of the Jacobian matrix requires to express all variables that are changing due to differential equations. In batch reactor systems, as shown in the previous section, relevant variables are given by concentrations of reagents, as well as the reactor temperature. Hence it is necessary to know how the concentration of each reagent changes.

To derive the divergence, a sample reaction network with $M$ reactions is considered for which the general form of the divergence is derived. The reaction network is given by a set of parallel reactions with two reacting components resulting in a single product. This assumption is used for clarity of the derivation, but does not limit the validity of this derivation for different reaction types. The reaction network considered for the derivation in this work is given by the following expressions: 


$$
\begin{gathered}
\nu_{\mathrm{A}, 1} \mathrm{~A}+\nu_{\mathrm{B}, 1} \mathrm{~B} \rightarrow \mathrm{C} \\
\vdots \\
\nu_{\mathrm{A}, i} \mathrm{~A}+\nu_{\mathrm{D}, i} \mathrm{D} \rightarrow \mathrm{E} \\
\vdots \\
\nu_{\mathrm{A}, M} \mathrm{~A}+\nu_{\mathrm{G}, M} \mathrm{G} \rightarrow \mathrm{H} \\
i=1,2, \ldots, M
\end{gathered}
$$

where the reactions follow an Arrhenius expression according to Equation (2.13). The reaction rates are given by:

$$
\begin{gathered}
r_{1}=k_{0,1} \exp \left(\frac{-E_{a, 1}}{R T_{\mathrm{R}}}\right)[\mathrm{A}]^{n_{\mathrm{A}, 1}}[\mathrm{~B}]^{n_{\mathrm{B}, 1}} \\
\vdots \\
r_{i}=k_{0, i} \exp \left(\frac{-E_{a, i}}{R T_{\mathrm{R}}}\right)[\mathrm{A}]^{n_{\mathrm{A}, i}}[\mathrm{D}]^{n_{\mathrm{D}, i}} \\
\vdots \\
r_{M}=k_{0, M} \exp \left(\frac{-E_{a, M}}{R T_{\mathrm{R}}}\right)[\mathrm{A}]^{n_{\mathrm{A}, M}}[\mathrm{G}]^{n_{\mathrm{G}, M}}
\end{gathered}
$$

$$
i=1,2, \ldots, M
$$

where as for Equation (2.16) index $i$ represents the $i^{\text {th }}$ reaction within the $M$ reactions present.

The divergence of the Jacobian for this reaction network, occurring in a batch reactor with an energy balance according to Equation (2.14), is given by the following equation: 


$$
\begin{aligned}
& \operatorname{div}[\mathbf{J}] \cdot t_{\mathrm{ref}}=-\nu_{\mathrm{A}, 1} n_{\mathrm{A}, 1} k_{0,1} \exp \left(-\frac{E_{a, 1}}{R T_{\mathrm{R}}}\right)[\mathrm{A}]^{n_{\mathrm{A}, 1}-1}[\mathrm{~B}]^{n_{\mathrm{B}, 1}} \\
& -\nu_{\mathrm{B}, 1} n_{\mathrm{B}, 1} k_{0,1} \exp \left(-\frac{E_{a, 1}}{R T_{\mathrm{R}}}\right)[\mathrm{A}]^{n_{\mathrm{A}, 1}}[\mathrm{~B}]^{n_{\mathrm{B}, 1}-1} \\
& -\nu_{\mathrm{A}, i} n_{\mathrm{A}, i} k_{0, i} \exp \left(-\frac{E_{a, i}}{R T_{\mathrm{R}}}\right)[\mathrm{A}]^{n_{\mathrm{A}, i}-1}[\mathrm{D}]^{n_{\mathrm{D}, i}} \\
& -\nu_{\mathrm{D}, i} n_{\mathrm{D}, i} k_{0, i} \exp \left(-\frac{E_{a, i}}{R T_{\mathrm{R}}}\right)[\mathrm{A}]^{n_{\mathrm{A}, i}}[\mathrm{D}]^{n_{\mathrm{D}, i}-1} \\
& \vdots \\
& -\nu_{\mathrm{A}, M} n_{\mathrm{A}, M} k_{0, M} \exp \left(-\frac{E_{a, M}}{R T_{\mathrm{R}}}\right)[\mathrm{A}]^{n_{\mathrm{A}, M}-1}[\mathrm{G}]^{n_{\mathrm{G}, M}} \\
& -\nu_{\mathrm{G}, M} n_{\mathrm{G}, M} k_{0, M} \exp \left(-\frac{E_{a, M}}{R T_{\mathrm{R}}}\right)[\mathrm{A}]^{n_{\mathrm{A}, M}}[\mathrm{G}]^{n_{\mathrm{G}, M}-1} \\
& +\frac{1}{\rho C_{p} V_{\mathrm{R}}} \text {. } \\
& {\left[\frac{E_{a, 1}}{R T_{\mathrm{R}}^{2}} k_{0,1} \exp \left(-\frac{E_{a, 1}}{R T_{\mathrm{R}}}\right)[\mathrm{A}]^{n_{\mathrm{A}, 1}}[\mathrm{~B}]^{n_{\mathrm{B}, 1}}\left(-\Delta H_{r, 1}\right) V_{\mathrm{R}}\right.} \\
& +\frac{E_{a, i}}{R T_{\mathrm{R}}^{2}} k_{0, i} \exp \left(-\frac{E_{a, i}}{R T_{\mathrm{R}}}\right)[\mathrm{A}]^{n_{\mathrm{A}, i}}[\mathrm{D}]^{n_{\mathrm{D}, i}}\left(-\Delta H_{r, i}\right) V_{\mathrm{R}} \\
& \text { : } \\
& \left.+\frac{E_{a, M}}{R T_{\mathrm{R}}^{2}} k_{0, M} \exp \left(-\frac{E_{a, M}}{R T_{\mathrm{R}}}\right)[\mathrm{A}]^{n_{\mathrm{A}, M}}[\mathrm{G}]^{n_{\mathrm{G}, M}}\left(-\Delta H_{r, M}\right) V_{\mathrm{R}}-U A\right]
\end{aligned}
$$

The expression given in Equation (2.18) can be further generalized to give the following expression: 


$$
\begin{aligned}
\operatorname{div}[\mathbf{J}] t_{\mathrm{ref}}= & -\left(\nu_{\mathrm{A}, 1} n_{\mathrm{A}, 1} \mathrm{Da}_{\mathrm{A}, 1}+\nu_{\mathrm{B}, 1} n_{\mathrm{B}, 1} \mathrm{Da}_{\mathrm{B}, 1}\right) \exp \left(-\gamma_{1}\right) \\
& -\left(\nu_{\mathrm{A}, i} n_{\mathrm{A}, i} \mathrm{Da}_{\mathrm{A}, i}+\nu_{\mathrm{D}, i} n_{\mathrm{D}, i} \operatorname{Da}_{\mathrm{D}, i}\right) \exp \left(-\gamma_{i}\right) \\
\vdots & \\
& -\left(\nu_{\mathrm{A}, M} n_{\mathrm{A}, M} \mathrm{Da}_{\mathrm{A}, M}+\nu_{\mathrm{G}, M} n_{\mathrm{G}, M} \mathrm{Da}_{\mathrm{G}, M}\right) \exp \left(-\gamma_{M}\right) \\
& +\sum_{i=1}^{M}\left(B_{i} \gamma_{i} \mathrm{Da}_{\mathrm{A}, i} \exp \left(-\gamma_{i}\right)\right)-\mathrm{St}
\end{aligned}
$$

In the general case the components so far given as $\mathrm{A}, \mathrm{B}, \mathrm{G}$ and $\mathrm{H}$ in Equation (2.16), are denoted by index $j$. The resultant Damköhler number for reaction $i$ with $N$ number of reagents, as given in Equation (2.8) for the single reaction, is given by:

$$
\operatorname{Da}_{\mathrm{res}, i}=\sum_{j=1}^{N}\left(\nu_{j, i} n_{j, i} \mathrm{Da}_{j, i}\right), \quad i=1,2, \ldots, M
$$

The resultant Damköhler number for reaction $i, \mathrm{Da}_{\mathrm{res}, i}$, is required when analyzing the effect of the Arrhenius factor $k_{0, i}$ on the divergence of the Jacobian.

The divergence of the Jacobian for a multi-reaction system can be generalized for $M$ reactions with a total of $N$ reagents, each with their respective reaction orders and stoichiometric coefficients. When looking at Equation (2.19), the generalized form of the divergence is given by the following equation:

$$
\operatorname{div}[\mathbf{J}] \cdot t_{\mathrm{ref}}=\sum_{i=1}^{M}\left(\left[\sum_{j=1}^{N}\left(-\nu_{j, i} n_{j, i} \mathrm{Da}_{j, i}\right)+B_{i} \gamma_{i} \mathrm{Da}_{l, i}\right] \exp \left(-\gamma_{i}\right)\right)-\mathrm{St}
$$

where $\mathrm{Da}_{l, i}$ represents a Damköhler number which is not zero for the $i^{\text {th }}$ reaction. Not every reactant present in the system will contribute towards reaction $i$. Hence it is necessary to choose a reagent $l$ that does not have zero order for reaction $i$ resulting in $\mathrm{Da}_{l, i}$. The expression given in Equation (2.21) is used for the further generalization of thermal stability criterion $\mathcal{K}$.

From Equation (2.21) it can be seen that every reaction $i$ contributes to the total divergence of the system. Solely the Stanton number, St, appears once as this represents the cooling of the reactor. The individual part of the divergence of the Jacobian related to each 
reaction $i$, denoted $\mathcal{D}_{i}$, is given by:

$$
\mathcal{D}_{i}=\left[\sum_{j=1}^{N}\left(-\nu_{j, i} n_{j, i} \mathrm{Da}_{j, i}\right)+B_{i} \gamma_{i} \mathrm{Da}_{l, i}\right] \exp \left(-\gamma_{i}\right)
$$

Using Equations (2.21) and (2.22), the final form of the total divergence of the Jacobian for a multiple reaction system can be summarized by the following:

$$
\operatorname{div}[\mathbf{J}] \cdot t_{\mathrm{ref}}=\sum_{i=1}^{M} \mathcal{D}_{i}-\mathrm{St}
$$

Equation (2.23) will be used in the generalization of thermal stability criterion $\mathcal{K}$.

\subsubsection{Expression for criterion $\mathcal{K}$ for multiple reactions}

The thermal stability criterion for a multi-reaction system is given by the same expression as for a single reaction system, given by Equation (2.3):

$$
\mathcal{K}^{(s)}=\operatorname{div}\left[\mathbf{J}^{(s)}\right]-\left|\mathcal{E}^{(s)}\right|
$$

where it is now necessary to find an expression for $\mathcal{E}$ for multiple reaction systems.

The generalized expression for $\mathcal{E}^{(s)}$ is given by Equation (2.9):

$$
\mathcal{E}^{(s)}=\operatorname{div}\left[\mathbf{J}^{(s-1)}\right]+\operatorname{div}\left[\mathbf{J}^{(s-1)}\right] \cdot \operatorname{d} \ln \left(\operatorname{div}\left[\mathbf{J}^{(s)}\right]\right)
$$

The generalized form of the divergence was derived in Equation (2.23). Hence it is now necessary to find an expression for $\mathrm{d} \ln \left(\operatorname{div}\left[\mathbf{J}^{(s)}\right]\right)$ within Equation (2.9) given above.

From Equation (2.23) it is true in general that $\left(\operatorname{div}[\mathbf{J}] \cdot t_{\text {ref }}\right)$ is a function of $\mathcal{D}_{i}$ and $\mathrm{St}$ for a total of $M$ reactions. Therefore Equation (2.23) is given by the following:

$$
\operatorname{div}[\mathbf{J}] \cdot t_{\mathrm{ref}}=f\left(\mathcal{D}_{i}, \mathrm{St}\right), \quad i=1,2, \ldots, M
$$

The form of the total divergence of the Jacobian, $\operatorname{div}[\mathbf{J}] \cdot t_{\text {ref }}$, now allows a total derivative to be carried out:

$$
\mathrm{d}\left(\operatorname{div}[\mathbf{J}] \cdot t_{\mathrm{ref}}\right)=\sum_{i=1}^{M} \frac{\partial\left(\operatorname{div}[\mathbf{J}] \cdot t_{\mathrm{ref}}\right)}{\partial\left(\mathcal{D}_{i}\right)} \mathrm{d}\left(\mathcal{D}_{i}\right)+\frac{\partial\left(\operatorname{div}[\mathbf{J}] \cdot t_{\mathrm{ref}}\right)}{\partial(\mathrm{St})} \mathrm{d}(\mathrm{St})
$$

In order to reformulate the expression given in Equation (2.26) into the correct form, the 
differential of a logarithm is introduced:

$$
\mathrm{d} \ln x^{(s)}=\frac{\mathrm{d} x^{(s)}}{x^{(s-1)}}=\lim _{\Delta x^{(s)} \rightarrow 0} \frac{\Delta x^{(s)}}{x^{(s-1)}} \approx \frac{x^{(s)}-x^{(s-1)}}{x^{(s-1)}}
$$

Using the expression for the differential of a logarithm, Equation (2.26) can be reformulated to give the following expression including logarithmic terms:

$$
\begin{aligned}
\mathrm{d}\left(\operatorname{div}[\mathbf{J}] \cdot t_{\mathrm{ref}}\right)= & \sum_{i=1}^{M} \mathcal{D}_{i} \frac{\partial\left(\operatorname{div}[\mathbf{J}] \cdot t_{\mathrm{ref}}\right)}{\partial\left(\mathcal{D}_{i}\right)} \mathrm{d}\left[\ln \left(\mathcal{D}_{i}\right)\right] \\
& +\left(\operatorname{div}[\mathbf{J}] \cdot t_{\mathrm{ref}}\right) \cdot \frac{\partial\left[\ln \left(\operatorname{div}[\mathbf{J}] \cdot t_{\mathrm{ref}}\right)\right]}{\partial \ln (\mathrm{St})} \mathrm{d}[\ln (\mathrm{St})]
\end{aligned}
$$

$\mathcal{D}_{i}$ is the part of the divergence which is only influenced by each individual reaction. In Section 2.3 it is shown how the divergence of the Jacobian for a single reaction can be used to find an expression for $\mathcal{E}$. Similarly, the summation of each individual contribution for each reaction will lead to the generalized expression of $\mathcal{E}$ for a multiple reaction system. To find such an expression, it is required to find an equation for $\mathrm{d}\left[\ln \left(\mathcal{D}_{i}\right)\right]$ within Equation $(2.28)$. From Equation (2.22) the function for $\mathcal{D}_{i}$ is given by:

$$
\mathcal{D}_{i}=f\left(B_{i}, \gamma_{i}, \mathrm{Da}_{\mathrm{res}, i}\right)
$$

The total differential of $\mathrm{d}\left[\ln \left(\mathcal{D}_{i}\right)\right]$ given in Equation $(2.28)$ is derived in the following manner:

$$
\begin{aligned}
\mathrm{d}\left[\ln \left(\mathcal{D}_{i}\right)\right] & =\frac{\partial \ln \left(\mathcal{D}_{i}\right)}{\partial \ln \left(B_{i}\right)} \mathrm{d} \ln \left(B_{i}\right)+\frac{\partial \ln \left(\mathcal{D}_{i}\right)}{\partial \ln \left(\gamma_{i}\right)} \mathrm{d} \ln \left(\gamma_{i}\right)+\frac{\partial \ln \left(\mathcal{D}_{i}\right)}{\partial \ln \left(\mathrm{Da}_{\mathrm{res}, i}\right)} \mathrm{d} \ln \left(\mathrm{Da}_{\mathrm{res}, i}\right) \\
\mathrm{d}\left[\ln \left(\mathcal{D}_{i}\right)\right] & =m_{B} \mathrm{~d} \ln \left(B_{i}\right)+m_{\gamma} \mathrm{d} \ln \left(\gamma_{i}\right)+m_{\text {Dares }} \mathrm{d} \ln \left(\mathrm{Da}_{\mathrm{res}, i}\right)
\end{aligned}
$$

The Stanton number coefficient does not appear in Equation (2.30b), as each individual reaction does not have an effect on this dimensionless variable. Therefore this is taken into account separately in the expression for the total divergence of the Jacobian. As was the case for a single reaction in Section 2.3, the gradient coefficient for the Stanton number is given by:

$$
\frac{\partial \ln \left(\operatorname{div}[\mathbf{J}] \cdot t_{\mathrm{ref}}\right)}{\partial \ln (\mathrm{St})}=m_{\mathrm{St}}
$$

The value of coefficients $m_{B}, m_{\gamma}, m_{\mathrm{Da}_{\mathrm{res}}}$, and $m_{\mathrm{St}}$ were derived in Kähm and Vassiliadis 
(2018c) for a wide variety of possible reaction kinetics for a single reaction. Hence it is tested if the values found can be applied for a general reaction $i$ within a reaction network. The trajectory of each individual dimensionless variable $B_{i}, \gamma_{i}$ and $\mathrm{Da}_{\mathrm{res}, i}$ will be different for each reaction and needs to be evaluated separately.

It is further noted from Equation (2.23) that:

$$
\frac{\partial\left(\operatorname{div}[\mathbf{J}] \cdot t_{\mathrm{ref}}\right)}{\partial\left(\mathcal{D}_{i}\right)}=1 \quad i=1,2, \ldots, M
$$

This result, together with the results from Equations (2.30b) and (2.31), allows the simplification of the total divergence of a general reaction system, given in Equation (2.28):

$$
\begin{aligned}
\operatorname{div}[\mathbf{J}] \cdot \mathrm{d} \ln (\operatorname{div}[\mathbf{J}])= & \sum_{i=1}^{M} \mathcal{D}_{i}\left[m_{B} \mathrm{~d} \ln \left(B_{i}\right)+m_{\gamma} \mathrm{d} \ln \left(\gamma_{i}\right)+m_{\text {Dares }_{\text {res }}} \mathrm{d} \ln \left(\mathrm{Da}_{\mathrm{res}, i}\right)\right] \\
& +\operatorname{div}[\mathbf{J}] \cdot m_{\mathrm{St}} \mathrm{d}[\ln (\mathrm{St})]
\end{aligned}
$$

In Equation (2.33) several interesting features can be observed: each reaction contributes towards the total divergence according to its individual divergence $\mathcal{D}_{i}$, therefore giving a weighting for the thermal runaway behavior. This means that if a reaction is very slow or produces very little heat, its value for $\mathcal{D}_{i}$ is small and hence its contribution to the thermal runaway is small, too. The Stanton number appears separately, as discussed above. The contribution of the Stanton number is the same, no matter how many reactions take place. This is intuitive, as the Stanton number only depends on the cooling jacket properties, and not the reaction kinetics within the reactor.

The final step of the derivation requires to find an expression for $\mathcal{E}$. As was the case in Section 2.3, it is necessary to find an expression for $\mathcal{E}^{(s)}$ at time step $(s)$ as a function of each individual contribution towards the total divergence in time step $(s-1), \mathcal{D}_{i}^{(s-1)}$, and the dimensionless variables at time steps $(s-1)$ and $(s)$. For multiple reactions the function for $\mathcal{E}^{(s)}$ is given by:

$$
\mathcal{E}^{(s)}=f\left(\mathcal{D}_{i}^{(s-1)}, B_{i}^{(s)}, B_{i}^{(s-1)}, \gamma_{i}^{(s)}, \gamma_{i}^{(s-1)}, \mathrm{Da}_{\mathrm{res}, i}^{(s)}, \mathrm{Da}_{\mathrm{res}, i}^{(s-1)}, \mathrm{St}^{(s)}, \mathrm{St}^{(s-1)}\right), \quad i=1,2, \ldots, M
$$

where $\mathcal{D}_{i}^{(s-1)}$ is a function of all dimensionless groups mentioned in time step $(s-1)$.

From Equations (2.9) and (2.33) the correction function $\mathcal{E}^{(s)}$ at time step $(s)$ can be found: 


$$
\begin{aligned}
\mathcal{E}^{(s)}= & \operatorname{div}\left[\mathbf{J}^{(s-1)}\right]+\sum_{i=1}^{M} \mathcal{D}_{i}^{(s-1)}\left[m_{B} \frac{B_{i}^{(s)}-B_{i}^{(s-1)}}{B_{i}^{(s-1)}}+m_{\gamma} \frac{\gamma_{i}^{(s)}-\gamma_{i}^{(s-1)}}{\gamma_{i}^{(s-1)}}+m_{\mathrm{Da}_{\mathrm{res}}} \frac{\operatorname{Da}_{\mathrm{res}, i}^{(s)}-\mathrm{Da}_{\mathrm{res}, i}^{(s-1)}}{\operatorname{Da}_{\mathrm{res}, i}^{(s-1)}}\right] \\
& +\operatorname{div}\left[\mathbf{J}^{(s-1)}\right] \cdot m_{\mathrm{St}} \frac{\mathrm{St}^{(s)}-\mathrm{St}^{(s-1)}}{\mathrm{St}^{(s-1)}}
\end{aligned}
$$

which includes all the variables as required in Equation (2.34).

Now that the necessary form of $\mathcal{E}^{(s)}$ is derived, thermal stability criterion $\mathcal{K}$ can be evaluated according to the definition given in Equation (2.3):

$$
\begin{aligned}
\mathcal{K}^{(s)}= & \operatorname{div}\left[\mathbf{J}^{(s)}\right]-\mid \operatorname{div}\left[\mathbf{J}^{(s-1)}\right]+\sum_{i=1}^{M} \mathcal{D}_{i}^{(s-1)}\left[m_{B} \frac{B_{i}^{(s)}-B_{i}^{(s-1)}}{B_{i}^{(s-1)}}+m_{\gamma} \frac{\gamma_{i}^{(s)}-\gamma_{i}^{(s-1)}}{\gamma_{i}^{(s-1)}}\right. \\
& \left.+m_{\text {Dares }_{\text {res }}} \frac{\operatorname{Da}_{\text {res }, i}^{(s)}-\mathrm{Da}_{\mathrm{res}, i}^{(s-1)}}{\operatorname{Da}_{\mathrm{res}, i}^{(s-1)}}\right]+\operatorname{div}\left[\mathbf{J}^{(s-1)}\right] \cdot m_{\mathrm{St}} \frac{\mathrm{St}^{(s)}-\mathrm{St}^{(s-1)}}{\mathrm{St}^{(s-1)}} \mid
\end{aligned}
$$

This concludes the generalization of thermal stability criterion $\mathcal{K}$ for multiple reaction systems. It can clearly be seen that the stability criterion $\mathcal{K}$ for multiple reaction systems, given in Equation (2.36), is of similar form as Equation (2.11) for a single chemical reaction. The derived equation for multiple reaction systems adds the contribution of each individual reaction towards the total divergence of the Jacobian. One of the goals of this work is to validate the applicability of the gradient coefficients $m_{B}, m_{\gamma}, m_{\mathrm{Da}_{\mathrm{res}}}$ and $m_{\mathrm{St}}$ to evaluate the thermal stability of batch processes with multiple reactions. How well this form evaluates the stability of batch systems is examined in Section 4.

\section{Batch reactor model}

The batch reactor model underlying the simulations in this work is outlined in this section. All assumptions employed, equations used, and the PI controller applied to the system are discussed in detail. The results of using this reactor model are shown in Section 4 with PI control and in Section 5 with MPC.

\subsection{Batch reactor parameters}

Batch reactors are a major part of the chemical industry. This type of reactor enables to run processes in a flexible manner, because operating conditions can be changed during the process to reach product specifications.

To clearly identify the important parts which lead to the reactor model presented in this section, a flow sheet of the batch reactor used in the simulations is shown in Figure 1. 


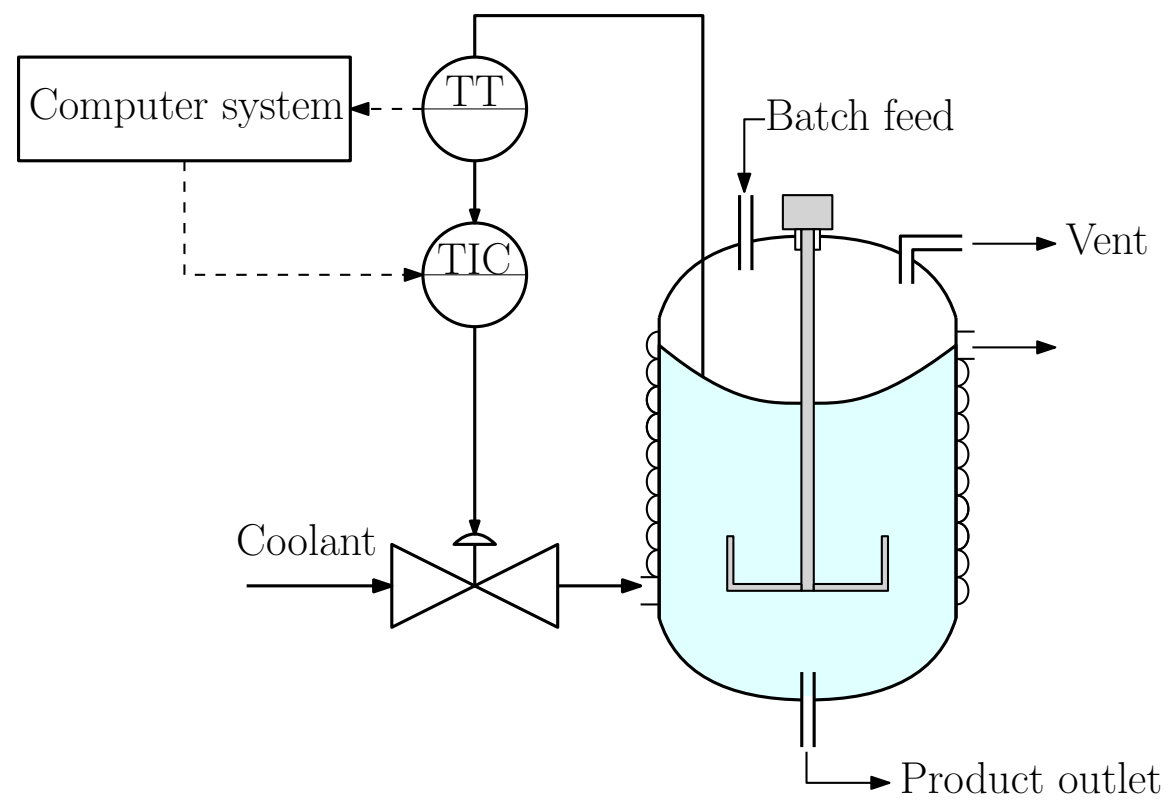

Figure 1: Batch reactor diagram for simulated systems.

Before the start of the reaction, the reactants are added into the reactor through the batch feed. Then the contents are heated up until the required initial temperature is reached by running steam through the cooling jacket. This heating up process is not included in the simulations, but the simulations start at the initial temperatures after the heating procedure has completed. The temperature information is transmitted by a Temperature-Transmitter (TT) to a computer system which control the set-point temperature for the TemperatureIntegrated-Controller (TIC) controller. The computer system can include an MPC algorithm, or a PI controller. Once the final conversion is achieved, the products are released through the product outlet in Figure 1.

The mixing of the reacting mixture is achieved by a Rushton impeller (Paul et al., 2004). In all models strong mixing is assumed, reaching a Reynolds number for the impeller of approximately $10^{6}$. Highly turbulent flow within the reactor leads to the assumption of uniform physical properties in the radial and axial directions of the batch reactor vessel. The heat generated by the stirring action in the reactor is negligible in comparison to the heat generation by the exothermic reactions. Hence, this effect of the stirrer is omitted form all simulations carried out in this work.

To cover a variety of dynamic behaviors, different reactor parameters are used for different processes. The data of the different reactor settings are shown in Table 1.

The contents within batch reactors are filled up to $80 \%$ of the total volume to leave space for stirred contents and possible foam formation. Hence, the values of $V_{\mathrm{R}}$ shown in Table 1 represent the volume of the reagents and not the volume of the whole reactor. 
Table 1: Batch reactor parameters for the processes considered.

\begin{tabular}{ccccc}
\hline Process & $V_{\mathrm{R}}\left[\mathrm{m}^{3}\right]$ & $V_{\mathrm{C}}\left[\mathrm{m}^{3}\right]$ & $A\left[\mathrm{~m}^{2}\right]$ & $q_{\mathrm{C}, \text { in }}\left[\mathrm{m}^{3} \mathrm{~s}^{-1}\right]$ \\
\hline $\mathrm{P}_{1}^{1}-\mathrm{P}_{4}^{1}$ & 32 & 2.0 & 49.1 & 0.060 \\
$\mathrm{P}_{5}^{1}-\mathrm{P}_{6}^{1}$ & 25 & 1.7 & 42.2 & 0.051 \\
$\mathrm{P}_{1}^{2}-\mathrm{P}_{4}^{2}$ & 20 & 1.4 & 35.8 & 0.043 \\
Nitration of toluene & 8 & 0.5 & 20.0 & 0.023 \\
\hline
\end{tabular}

The heat transfer coefficient for the heat transfer between the cooling jacket and the reactor contents $U$ depends on the physical properties of the coolant and the reacting mixture, as well as the flow intensity on both sides (Sinnot, 2005). As was shown when describing the stirrer type used, turbulent flow is present within the reactor. Hence, the major contribution to the change in $U$ are the physical properties of the reacting mixture and the cooling flow rate.

The temperature within batch reactors can be controlled in several different ways. Proportional Integral (PI) control is most commonly found in industry for this purpose (Winde, 2009). In this work, PI control is used to examine how well the generalization of stability criterion $\mathcal{K}$ works for multiple reaction systems. The mathematical description of a PI controller is shown in detail in Equation (2.12) in Kähm and Vassiliadis (2018d). The proportional constant $K_{P}$ and integral constant $\tau_{I}$ define how the PI controller behaves for the process, and are set to $K_{P}=10 \mathrm{~m}^{3} \mathrm{~K}^{-1} \mathrm{~s}^{-1}$ and $\tau_{I}=1000 \mathrm{~K} \mathrm{~s}^{2} \mathrm{~m}^{-3}$. The purpose of the PI controller used in this work is to examine when each batch process becomes unstable if the set-point temperature is set too high.

The systems were simulated using ode15s (Shampine et al., 1999) within MATLAB ${ }^{\mathrm{TM}}$, which uses an adjusted time step Runge-Kutta method. MATLAB ${ }^{\mathrm{TM}}$ was used due to its simplicity of developing code. The SQP optimization algorithm within MATLAB ${ }^{\mathrm{TM}}$ is used. The optimization algorithm does not have to guarantee global optimality to be useful for nonlinear MPC formulations (Durand and Christofides, 2016; Ellis and Christofides, 2015; Santos et al., 1995). All simulations shown in this paper were carried out on an HP EliteDesk 800 G2 Desktop Mini PC with an Intel@ Core i5-65000 processor with $3.20 \mathrm{GHz}$ and 16.0 GB RAM, running on Windows 7 Enterprise.

\subsection{Reaction kinetics}

The reactions analyzed in this work occur in a homogeneous liquid solution and are assumed to be irreversible. A total of three different reaction schemes are considered in this work. Reaction scheme 1 consists of 4 chemical reactions occurring in parallel. These 4 reactions are included within reaction scheme 2 , for which two more reactions are added. 
Hence reaction scheme 2 results in 6 reactions occurring in parallel. The final reaction scheme is the nitration of toluene commonly found in industry.

\subsubsection{Reaction scheme 1}

The first reaction scheme consists of parallel competing reactions given by the following expressions:

$$
\begin{aligned}
& \nu_{\mathrm{A},{ }_{1}} \mathrm{~A}+\nu_{\mathrm{B}, 1} \mathrm{~B} \rightarrow \mathrm{C} \\
& \nu_{\mathrm{A},{ }_{2}} \mathrm{~A}+\nu_{\mathrm{C},{ }_{2}} \mathrm{C} \rightarrow \mathrm{D} \\
& \nu_{\mathrm{A},{ }_{3}} \mathrm{~A}+\nu_{\mathrm{B},{ }_{3}} \mathrm{~B} \rightarrow \mathrm{E} \\
& \nu_{\mathrm{A},{ }_{4}} \mathrm{~A}+\nu_{\mathrm{E}, 4} \mathrm{E} \rightarrow \mathrm{F}
\end{aligned}
$$

The reaction rates are given by Arrhenius expressions (Davis and Davis, 2003) given by:

$$
\begin{aligned}
& r_{1}=k_{0,1} \exp \left(-\frac{E_{a, 1}}{R T_{\mathrm{R}}}\right)[\mathrm{A}]^{n_{\mathrm{A}, 1}}[\mathrm{~B}]^{n_{\mathrm{B}, 1}} \\
& r_{2}=k_{0,2} \exp \left(-\frac{E_{a, 2}}{R T_{\mathrm{R}}}\right)[\mathrm{A}]^{n_{\mathrm{A}, 2}}[\mathrm{C}]^{n_{\mathrm{C}, 2}} \\
& r_{3}=k_{0,3} \exp \left(-\frac{E_{a, 3}}{R T_{\mathrm{R}}}\right)[\mathrm{A}]^{n_{\mathrm{A}, 3}}[\mathrm{~B}]^{n_{\mathrm{B}, 3}} \\
& r_{4}=k_{0,4} \exp \left(-\frac{E_{a, 4}}{R T_{\mathrm{R}}}\right)[\mathrm{A}]^{n_{\mathrm{A}, 4}}[\mathrm{E}]^{n_{\mathrm{E}, 4}}
\end{aligned}
$$

The reaction rate giving rise to $r_{i}$ is called reaction $i$ hereafter. Hence reactions 1 and 2 are described by the rate equations given for $r_{1}$ and $r_{2}$ in Equations (3.2a) and (3.2b), respectively. Similarly, reactions 3 and 4 are described by the expressions for $r_{3}$ and $r_{4}$ in Equations (3.2c) and (3.2d), respectively.

The processes are denoted by $\mathrm{P}_{1}^{1}-\mathrm{P}_{6}^{1}$ for processes 1 through 6 within reaction scheme 1 . For reactions 1 and 2, the data used for processes $\mathrm{P}_{1}^{1}-\mathrm{P}_{6}^{1}$ are summarized in the top section of Table 2 . 
Table 2: Process parameters for reactions 1 and 2, 3 and 4 for processes $\mathrm{P}_{1}^{1}-\mathrm{P}_{6}^{1}$, and reactions 5 and 6 for processes $\mathrm{P}_{1}^{2}-\mathrm{P}_{4}^{2}$.

\begin{tabular}{cccccccc}
\hline Process & $\nu_{\mathrm{A}, 1} ;$ & $\nu_{\mathrm{A}, 2} ;$ & $n_{\mathrm{A}, 1} ;$ & $n_{\mathrm{A}, 2} ;$ & $\begin{array}{c}k_{0,1} ; k_{0,2} \\
{\left[\mathrm{~m}^{3} \mathrm{~mol}^{-1} \mathrm{~s}^{-1}\right]}\end{array}$ & $\begin{array}{c}E_{a, 1} ; E_{a, 2} \\
{\left[\mathrm{~kJ} \mathrm{~mol}^{-1}\right]}\end{array}$ & $\begin{array}{c}\Delta H_{r, 1} ; \Delta H_{r, 2} \\
{\left[\mathrm{~kJ} \mathrm{~mol}^{-1}\right]}\end{array}$ \\
\hline$\nu_{1}^{1}$ & $1 ; 2$ & $2 ; 1$ & $1.5 ; 1.5$ & $1 ; 1$ & $100 ; 200$ & $60 ; 70$ & $-85 ;-75$ \\
$\mathrm{P}_{2}^{1}$ & $1 ; 3$ & $3 ; 1$ & $1 ; 2$ & $2 ; 1$ & $3 \times 10^{4} ; 2 \times 10^{4}$ & $80 ; 90$ & $-60 ;-55$ \\
$\mathrm{P}_{3}^{1}$ & $1 ; 2$ & $3 ; 2$ & $2 ; 2$ & $1.5 ; 1$ & $1.1 ; 0.7$ & $65 ; 75$ & $-90 ;+35$ \\
$\mathrm{P}_{4}^{1}$ & $1 ; 3$ & $1 ; 1$ & $1 ; 2.5$ & $1.5 ; 1.5$ & $2 \times 10^{4} ; 1.5 \times 10^{4}$ & $90 ; 92$ & $-100 ;-95$ \\
$\mathrm{P}_{5}^{1}$ & $1 ; 1$ & $2 ; 1$ & $3 ; 1.5$ & $2 ; 2.5$ & $2.1 ; 3.2$ & $58 ; 61$ & $-40 ;-50$ \\
$\mathrm{P}_{6}^{1}$ & $1 ; 2$ & $1 ; 1$ & $2 ; 1.5$ & $2 ; 1.5$ & $280 ; 170$ & $82 ; 84$ & $-55 ;-62$ \\
\hline Process & $\nu_{\mathrm{A}, 3} ;$ & $\nu_{\mathrm{A}, 4} ;$ & $n_{\mathrm{A}, 3} ;$ & $n_{\mathrm{A}, 4} ;$ & $k_{0,3} ; k_{0,4}$ & $E_{a, 3} ; E_{a, 4}$ & $\Delta H_{r, 3} ; \Delta H_{r, 4}$ \\
& $\nu_{\mathrm{B}, 3}$ & $\nu_{\mathrm{C}, 4}$ & $n_{\mathrm{B}, 3}$ & $n_{\mathrm{C}, 4}$ & {$\left[\mathrm{~m}^{3} \mathrm{~mol}^{-1} \mathrm{~s}^{-1}\right]$} & {$\left[\mathrm{kJ} \mathrm{mol}^{-1}\right]$} & $\left.[\mathrm{kJ} \mathrm{mol}]^{-1}\right]$ \\
\hline $\mathrm{P}_{1}^{1}$ & $1 ; 1$ & $1 ; 1$ & $1.5 ; 1$ & $1.5 ; 1$ & $100 ; 300$ & $83 ; 80$ & $-35 ;-45$ \\
$\mathrm{P}_{2}^{1}$ & $3 ; 1$ & $1 ; 3$ & $1 ; 1$ & $1 ; 1$ & $2 \times 10^{4} ; 3 \times 10^{4}$ & $71 ; 78$ & $-66 ;-48$ \\
$\mathrm{P}_{3}^{1}$ & $3 ; 2$ & $2 ; 1$ & $2 ; 2.5$ & $1.5 ; 1.5$ & $0.8 ; 1.9$ & $63 ; 75$ & $-120 ;-105$ \\
$\mathrm{P}_{4}^{1}$ & $1 ; 1$ & $1 ; 1$ & $2 ; 1.5$ & $2 ; 2.5$ & $1.5 \times 10^{4} ; 2 \times 10^{4}$ & $90 ; 92$ & $-95 ;-90$ \\
$\mathrm{P}_{5}^{1}$ & $2 ; 1$ & $1 ; 2$ & $1.5 ; 1$ & $2 ; 2$ & $8700 ; 9200$ & $73 ; 81$ & $-155 ;-165$ \\
$\mathrm{P}_{6}^{1}$ & $1 ; 1$ & $1 ; 1$ & $1.5 ; 1.5$ & $1.5 ; 3$ & $6 \times 10^{4} ; 4 \times 10^{4}$ & $87 ; 90$ & $-105 ;-125$ \\
\hline Process $^{1} \nu_{\mathrm{A}, 5} ;$ & $\nu_{\mathrm{A}, 6} ;$ & $n_{\mathrm{A}, 5} ;$ & $n_{\mathrm{A}, 6} ;$ & $k_{0,5} ; k_{0,6} \times 10^{-5}$ & $E_{a, 5} ; E_{a, 6}$ & $\Delta H_{r, 5} ; \Delta H_{r, 6}$ \\
& $\nu_{\mathrm{B}, 5}$ & $\nu_{\mathrm{C}, 6}$ & $n_{\mathrm{B}, 5}$ & $n_{\mathrm{C}, 6}$ & {$\left[\mathrm{~m}^{3} \mathrm{~mol}^{-1} \mathrm{~s}^{-1}\right]$} & {$[\mathrm{kJ} \mathrm{mol}]$} & {$\left[\mathrm{kJ} \mathrm{mol}{ }^{-1}\right]$} \\
\hline $\mathrm{P}_{1}^{2}$ & $2 ; 1$ & $1 ; 1$ & $1.5 ; 1$ & $1.5 ; 1.5$ & $150 ; 190$ & $93 ; 90$ & $-115 ;-90$ \\
$\mathrm{P}_{2}^{2}$ & $2 ; 1$ & $1 ; 3$ & $1 ; 1$ & $1 ; 2$ & $1.1 \times 10^{4} ; 8000$ & $91 ; 94$ & $-92 ;+40$ \\
$\mathrm{P}_{3}^{2}$ & $3 ; 2$ & $1 ; 2$ & $1.5 ; 1.5$ & $2 ; 2$ & $1.7 ; 1.3$ & $89 ; 92$ & $-125 ;-95$ \\
$\mathrm{P}_{4}^{2}$ & $1 ; 1$ & $1 ; 3$ & $2 ; 2.5$ & $1 ; 2.5$ & $1400 ; 1500$ & $87 ; 65$ & $-100 ;-75$ \\
\hline
\end{tabular}

The reaction data for reactions 3 and 4 for processes $\mathrm{P}_{1}^{1}-\mathrm{P}_{6}^{1}$ are given in the second section of Table 2 .

The initial concentrations of reagents $A$ and $B$ are given by $[A]_{0}=15.0 \mathrm{kmol} \mathrm{m}^{-3}$ and $[\mathrm{B}]_{0}=17.0 \mathrm{kmol} \mathrm{m}^{-3}$. Components $\mathrm{C}, \mathrm{D}$ and $\mathrm{E}$ are products of the initial reactions between reagents $\mathrm{A}$ and $\mathrm{B}$, Hence their initial concentrations are set to zero. Furthermore, it is seen in Table 2 that a large variation in system parameters is used in order to validate the generalized form of thermal stability criterion $\mathcal{K}$.

Reaction scheme 1 is the basis for reaction scheme 2 . Therefore the data for reactions 1 to 4 given in Table 2 are the same for reaction scheme 2. Furthermore, the initial concentrations given above are used also for all processes in reaction scheme 2 . 


\subsubsection{Reaction scheme 2}

The second reaction scheme considered in this work is composed of the parallel reactions shown in reaction scheme 1, given in Equation (3.2), as well as an additional set of reactions occurring in parallel. These reactions are given by:

$$
\begin{array}{r}
\nu_{\mathrm{A}, 5} \mathrm{~A}+\nu_{\mathrm{B}, 5} \mathrm{~B} \rightarrow \mathrm{G} \\
\nu_{\mathrm{A}, 6} \mathrm{~A}+\nu_{\mathrm{G}, 6} \mathrm{G} \rightarrow \mathrm{H}
\end{array}
$$

As for reaction scheme 1 , the reaction rates are dependent on the concentration of the respective reagents and their respective reaction order. The rate equations are hence given by:

$$
\begin{aligned}
& r_{5}=k_{0,5} \exp \left(-\frac{E_{a, 5}}{R T_{\mathrm{R}}}\right)[\mathrm{A}]^{n_{\mathrm{A}, 5}}[\mathrm{~B}]^{n_{\mathrm{B}, 5}} \\
& r_{6}=k_{0,6} \exp \left(-\frac{E_{a, 6}}{R T_{\mathrm{R}}}\right)[\mathrm{A}]^{n_{\mathrm{A}, 6}}[\mathrm{G}]^{n_{\mathrm{G}, 6}}
\end{aligned}
$$

The reaction rates given in Equations (3.4) are again expressed as Arrhenius relations (Davis and Davis, 2003). The reaction rates $r_{5}$ and $r_{6}$ given in Equation (3.4) correspond to reaction 5 and 6 within reaction scheme 2 . The data of the additional reactions are given in the bottom section of Table 2 .

The data given in Table 2 are the basis for all reactions occurring in reaction scheme 2 . A total of 6 reactions are present, hence representing a good test case in order to validate the generalization of thermal stability criterion $\mathcal{K}$. As was the case for products $\mathrm{C}$, D, and E within reaction scheme $1, \mathrm{G}$ and $\mathrm{H}$ are both products of the reaction system. Hence the initial concentrations of products $\mathrm{G}$ and $\mathrm{H}$ are set to zero.

\subsubsection{Industrial case study: Nitration of toluene}

The nitration of toluene is an industrially relevant reaction, which consists of both endothermic and exothermic reactions (Halder et al., 2008). Overall a net exothermic process is present, which is why thermal runaways can occur for this reaction system. The reaction is initiated by the formation of a nitronium ion $\left(\mathrm{NO}_{2}^{+}\right)$, followed by 3 parallel reactions with toluene: 


$$
\begin{array}{cl}
\mathrm{HNO}_{3}+\mathrm{H}_{2} \mathrm{SO}_{4} \rightarrow \mathrm{NO}_{2}^{+}+\mathrm{HSO}_{4}^{-}+\mathrm{H}_{2} \mathrm{O} & \text { Reaction (1) } \\
\mathrm{NO}_{2}^{+}+\mathrm{C}_{7} \mathrm{H}_{8}+\mathrm{H}_{2} \mathrm{O} \rightarrow \mathrm{O}-\mathrm{C}_{7} \mathrm{H}_{7} \mathrm{NO}_{2}+\mathrm{H}_{3} \mathrm{O}^{+} & \text {Reaction (2) } \\
\mathrm{NO}_{2}^{+}+\mathrm{C}_{7} \mathrm{H}_{8}+\mathrm{H}_{2} \mathrm{O} \rightarrow \mathrm{p}-\mathrm{C}_{7} \mathrm{H}_{7} \mathrm{NO}_{2}+\mathrm{H}_{3} \mathrm{O}^{+} & \text {Reaction (3) } \\
\mathrm{NO}_{2}^{+}+\mathrm{C}_{7} \mathrm{H}_{8}+\mathrm{H}_{2} \mathrm{O} \rightarrow \mathrm{m}-\mathrm{C}_{7} \mathrm{H}_{7} \mathrm{NO}_{2}+\mathrm{H}_{3} \mathrm{O}^{+} & \text {Reaction (4) }
\end{array}
$$

where the letters o-, p- and m- stand for ortho, para and meta positions, respectively, of the nitronium ion on toluene (Mawardi, 1982). The reactions in Equations (3.5) are referred to as reactions $(1)-(4)$ hereafter. The concentration of the nitronium ion and toluene influence each of reactions (2)-(4). From an engineering standpoint the energetics of reactions (2)-(4) is similar, which is why the reaction enthalpies for these reactions are assumed to be equal. This simplification is not possible for the reaction kinetics: as described in Mawardi (1982) the product of such a reaction will form a molar mixture of $60 \%$ ortho-, $37 \%$ para-, and $3 \%$ meta-nitrotoluene.

Each individual reaction can be described by Arrhenius rate expressions. The reaction rates are given by the following expressions:

$$
\begin{aligned}
& r_{1}=k_{0,1} \exp \left(\frac{-E_{a, 1}}{R T_{R}}\right)\left[\mathrm{HNO}_{3}\right]^{n_{1,1}}\left[\mathrm{H}_{2} \mathrm{SO}_{4}\right]^{n_{2,1}} \\
& r_{2}=k_{0,2} \exp \left(\frac{-E_{a, 2}}{R T_{R}}\right)\left[\mathrm{NO}_{2}^{+}\right]^{n_{1,2}}\left[\mathrm{C}_{7} \mathrm{H}_{8}\right]^{n_{2,2}} \\
& r_{3}=k_{0,3} \exp \left(\frac{-E_{a, 3}}{R T_{R}}\right)\left[\mathrm{NO}_{2}^{+}\right]^{n_{1,3}}\left[\mathrm{C}_{7} \mathrm{H}_{8}\right]^{n_{2,3}} \\
& r_{4}=k_{0,4} \exp \left(\frac{-E_{a, 4}}{R T_{R}}\right)\left[\mathrm{NO}_{2}^{+}\right]^{n_{1,4}}\left[\mathrm{C}_{7} \mathrm{H}_{8}\right]^{n_{2,4}}
\end{aligned}
$$

where $n_{1, i}$ and $n_{2, i}$ are orders of reaction with respect to each reagent for reaction $i$. Important to note is that each of reactions $(2)-(4)$ produce a $\mathrm{H}_{3} \mathrm{O}^{+}$ion, which will combine with $\mathrm{HSO}_{4}^{-}$ to form $\mathrm{H}_{2} \mathrm{SO}_{4}$. Hence the sulphuric acid in this reaction network acts as a catalyst. The data used for this reaction network are given in Table 3.

This reaction network includes both, an endothermic dissociation reaction (1) and the highly exothermic electrophilic substitution reactions $(2)-(4)$. Hence, this reaction system presents a challenge in order to keep the process under control. The initial concentrations of each reagent are given by $\left[\mathrm{HNO}_{3}\right]_{0}=6.0 \mathrm{kmol} \mathrm{m}^{-3},\left[\mathrm{H}_{2} \mathrm{SO}_{4}\right]_{0}=1.0 \mathrm{kmol} \mathrm{m}^{-3}$, and $\left[\mathrm{C}_{7} \mathrm{H}_{8}\right]_{0}=5.5 \mathrm{kmol} \mathrm{m}^{-3}$. These initial concentrations are used throughout all case studies for the nitration of toluene. The reactor dimensions for this system are given in Table 1. 
Table 3: Process parameters for the nitration of toluene reaction network (Chen et al., 2008; Luo and Chang, 1998; Mawardi, 1982; Sheats and Strachan, 1978).

\begin{tabular}{cccccc}
\hline $\begin{array}{c}\text { Reaction } \\
i\end{array}$ & $\begin{array}{c}k_{0, i} \\
{\left[\mathrm{~m}^{3} \mathrm{~mol}^{-1} \mathrm{~s}^{-1}\right]}\end{array}$ & $\begin{array}{c}E_{a, i} \\
{\left[\mathrm{kJmol}^{-1}\right]}\end{array}$ & $\begin{array}{c}\Delta H_{r, i} \\
{\left[\mathrm{kJmol}^{-1}\right]}\end{array}$ & $\begin{array}{c}n_{1, i} \\
{[-]}\end{array}$ & $\begin{array}{c}n_{2, i} \\
{[-]}\end{array}$ \\
\hline$(1)$ & $2.00 \times 10^{3}$ & 76.5 & +30.0 & 1.00 & 1.00 \\
$(2)$ & 109 & 12.5 & -122 & 2.27 & 0.293 \\
$(3)$ & 67.3 & 12.5 & -122 & 2.27 & 0.293 \\
$(4)$ & 5.46 & 12.5 & -122 & 2.27 & 0.293 \\
\hline
\end{tabular}

\subsection{Physical properties}

As each reaction proceeds, the physical properties can be subject to change according to composition and temperature. The accurate description of all physical properties is very complex and would exceed the scope of this work. Hence the changes in viscosity and specific heat capacity of the reaction mixture are estimated according to Hirschfelder et al. (1955) and Green and Perry (2008). The physical data used for all reagents, present in the 3 reaction schemes presented above, are given in Table 4.

Table 4: Physical properties of components for reaction schemes 1 and 2, and for the nitration of toluene.

\begin{tabular}{|c|c|c|c|c|}
\hline Physical property & $\rho\left[\mathrm{kg} \mathrm{m}^{-3}\right]$ & $\mu\left[\mathrm{Pas}^{-1}\right]$ & $C_{p}\left[\mathrm{~J} \mathrm{~kg}^{-1} \mathrm{~K}^{-1}\right]$ & $\lambda\left[\mathrm{W} \mathrm{m}^{-1} \mathrm{~K}^{-1}\right.$ \\
\hline \multicolumn{5}{|l|}{ Component } \\
\hline $\mathrm{A}$ & 911 & $1.00 \cdot 10^{-4}$ & 1100 & 0.300 \\
\hline $\mathrm{B}$ & 790 & $3.00 \cdot 10^{-4}$ & 950 & 0.250 \\
\hline $\mathrm{C}$ & 1200 & $9.00 \cdot 10^{-4}$ & 850 & 0.150 \\
\hline $\mathrm{D}$ & 1205 & $2.00 \cdot 10^{-4}$ & 4200 & 0.685 \\
\hline $\mathrm{E}$ & 810 & $1.00 \cdot 10^{-4}$ & 1250 & 0.400 \\
\hline $\mathrm{F}$ & 790 & $3.00 \cdot 10^{-4}$ & 950 & 0.250 \\
\hline $\mathrm{G}$ & 1000 & $10.0 \cdot 10^{-4}$ & 750 & 0.100 \\
\hline $\mathrm{H}$ & 1300 & $2.00 \cdot 10^{-4}$ & 2250 & 0.850 \\
\hline Toluene & 870 & $6.00 \cdot 10^{-4}$ & 1700 & 0.141 \\
\hline Mono-nitrotoluene mixture & 1160 & $2.00 \cdot 10^{-4}$ & 1500 & 0.150 \\
\hline $\mathrm{HNO}_{3} / \mathrm{H}_{2} \mathrm{SO}_{4} / \mathrm{H}_{2} \mathrm{O}$ mixture & 1430 & $2.90 \cdot 10^{-4}$ & 2600 & 0.540 \\
\hline
\end{tabular}

\section{Verification of stability criterion $\mathcal{K}$}

To test if the derivation shown in Section 2 works for reaction networks, reaction schemes 1 and 2 presented in Section 3.2 are considered as case studies. To verify that for each of these processes thermal stability criterion $\mathcal{K}$ gives a reliable prediction of system stability, a PI controller is used to make a stable system unstable. This is achieved by increasing the set-point temperature of the PI controller, which regulates the coolant flow rate. Once the new set-point temperature is reached, the PI controller will try to regain control over the 
batch process. If the heat generated is larger than the cooling capacity, the temperature of the system starts to increase in an uncontrollable manner.

To identify where exactly the stability of the batch process is lost, a second simulation for each process is carried out. For this second simulation, the set-point temperature is increased at the same point in time, but by a lower value. Hence a process which can be still be controlled is obtained in contrast to the one which exhibits thermal runaway behavior. This comparison of a stable and an unstable process can be used to identify at which point in time the stability is lost. The verification of thermal stability criterion $\mathcal{K}$ is consequently carried out by reading off the values obtained for $\mathcal{K}$ at which time stability is lost. This procedure is carried out for processes $\mathrm{P}_{1}^{1}-\mathrm{P}_{6}^{1}$ and $\mathrm{P}_{1}^{2}-\mathrm{P}_{4}^{2}$.

\subsection{Verification of criterion $\mathcal{K}$ for reaction scheme 1}

The generalized form of the thermal stability criterion was derived in Section 2.3. For reaction scheme 1 the specific expression for criterion $\mathcal{K}$ is given by the following:

$$
\begin{aligned}
\mathcal{K}^{(s)}= & \operatorname{div}\left[\mathbf{J}^{(s)}\right]-\mid \operatorname{div}\left[\mathbf{J}^{(s-1)}\right] \\
& +\mathcal{D}_{1}^{(s-1)}\left[m_{B} \frac{B_{1}^{(s)}-B_{1}^{(s-1)}}{B_{1}^{(s-1)}}+m_{\gamma} \frac{\gamma_{1}^{(s)}-\gamma_{1}^{(s-1)}}{\gamma_{1}^{(s-1)}}+m_{\mathrm{Da}_{\mathrm{res}}} \frac{\mathrm{Da}_{\text {res }, 1}^{(s)}-\mathrm{Da}_{\text {res }, 1}^{(s-1)}}{\operatorname{Da}_{\text {res }, 1}^{(s-1)}}\right] \\
& +\mathcal{D}_{2}^{(s-1)}\left[m_{B} \frac{B_{2}^{(s)}-B_{2}^{(s-1)}}{B_{2}^{(s-1)}}+m_{\gamma} \frac{\gamma_{2}^{(s)}-\gamma_{2}^{(s-1)}}{\gamma_{2}^{(s-1)}}+m_{\text {Dares }} \frac{\operatorname{Da}_{\text {res }, 2}^{(s)}-\mathrm{Da}_{\text {res }, 2}^{(s-1)}}{\operatorname{Da}_{\text {res }, 2}^{(s-1)}}\right] \\
& +\mathcal{D}_{3}^{(s-1)}\left[m_{B} \frac{B_{3}^{(s)}-B_{3}^{(s-1)}}{B_{3}^{(s-1)}}+m_{\gamma} \frac{\gamma_{3}^{(s)}-\gamma_{3}^{(s-1)}}{\gamma_{3}^{(s-1)}}+m_{\text {Dares }} \frac{\operatorname{Da}_{\text {res }, 3}^{(s)}-\operatorname{Da}_{\text {res }, 3}^{(s-1)}}{\operatorname{Da}_{\text {res }, 3}^{(s-1)}}\right] \\
& +\mathcal{D}_{4}^{(s-1)}\left[m_{B} \frac{B_{4}^{(s)}-B_{4}^{(s-1)}}{B_{4}^{(s-1)}}+m_{\gamma} \frac{\gamma_{4}^{(s)}-\gamma_{4}^{(s-1)}}{\gamma_{4}^{(s-1)}}+m_{\text {Dares }} \frac{\operatorname{Da}_{\text {res }, 4}^{(s)}-\operatorname{Da}_{\text {res }, 4}^{(s-1)}}{\operatorname{Da}_{\text {res }, 4}^{(s-1)}}\right] \\
& +\operatorname{div}\left[\mathbf{J}^{(s-1)}\right] m_{\mathrm{St}} \frac{\mathrm{St}^{(s)}-\mathrm{St}^{(s-1)}}{\mathrm{St}^{(s-1)}} \mid
\end{aligned}
$$

where the dimensionless numbers for each reaction are evaluated according to Equation (2.7), and $\mathcal{D}_{i}^{(s-1)}$ for each reaction $i$ is evaluated according to Equation (2.22). The gradient coefficients $m_{B}, m_{\gamma}, m_{\mathrm{Da} \text { res }}$, and $m_{\mathrm{St}}$ are given in Equation (2.12), which were found in (Kähm and Vassiliadis, 2018c). For clarity, the form of $\mathcal{D}_{1}^{(s-1)}$ in Equation (4.1) is given by the following expression:

$$
\mathcal{D}_{1}^{(s-1)}=\left[-\nu_{\mathrm{A}, 1} n_{\mathrm{A}, 1} \mathrm{Da}_{\mathrm{A}, 1}^{(s-1)}-\nu_{\mathrm{B}, 1} n_{\mathrm{B}, 1} \mathrm{Da}_{\mathrm{B}, 1}^{(s-1)}+\gamma_{1}^{(s-1)} B_{1}^{(s-1)} \mathrm{Da}_{\mathrm{B}, 1}^{(s-1)}\right] \exp \left(-\gamma_{1}^{(s-1)}\right)
$$




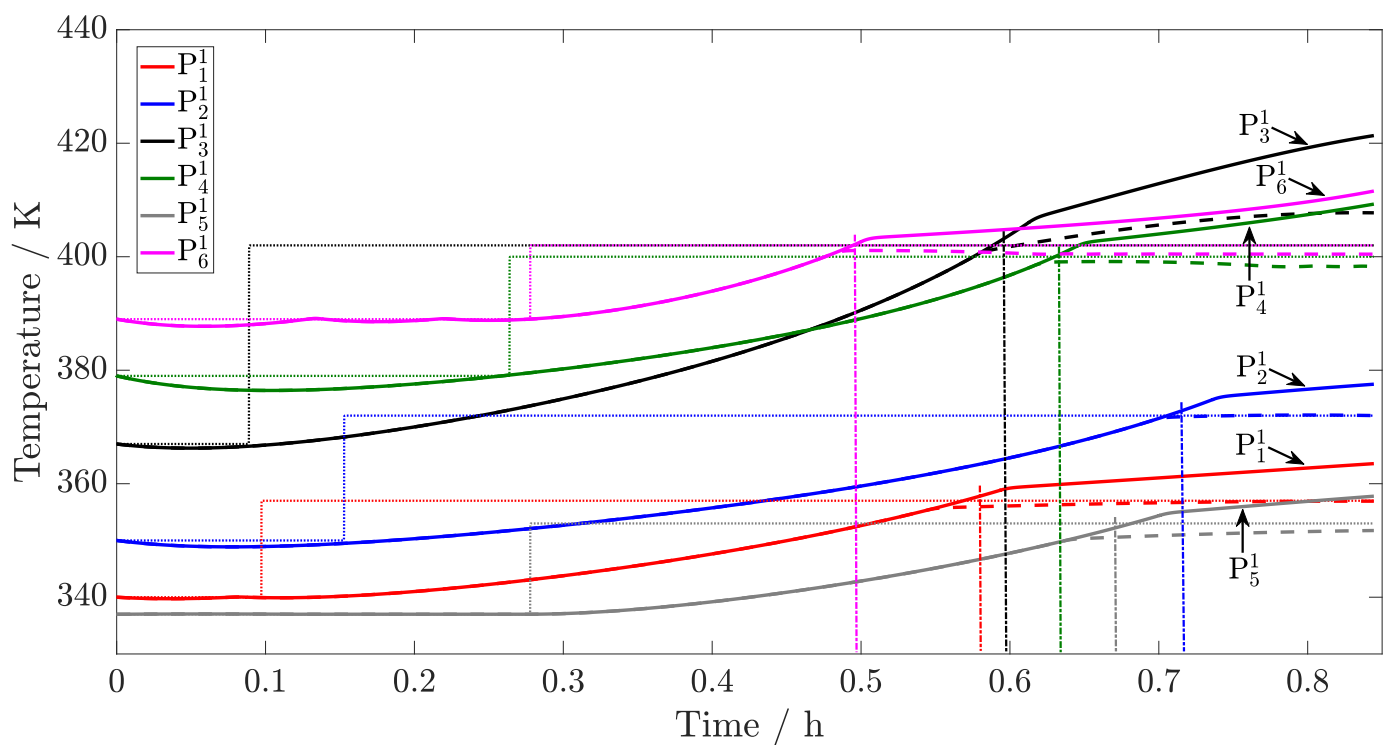

Figure 2: Temperature profiles for processes $\mathrm{P}_{1}^{1}-\mathrm{P}_{6}^{1}$. The dotted lines indicate the set-point temperatures for the PI controller. The dashed lines represent stable processes with lower increased set-point temperatures. The dash-dotted lines parallel to the y-axis indicate when stability is lost in the system.

In reaction scheme 1 a total of 4 reactions are present which overall are exothermic. The resulting temperature profiles for processes $\mathrm{P}_{1}^{1}-\mathrm{P}_{6}^{1}$ are shown in Figure 2.

In Figure 2 two simulations per process are shown. The solid lines indicate the simulations where each process becomes unstable after the increase in set-point temperature. As can be seen, the temperature continues to increase after reaching the dotted set-point temperature line, ultimately resulting in thermal runaway behavior. This is the case because the maximum coolant flow rate the PI controller can use is not enough to cool the system sufficiently.

The dashed lines represent the same processes, with a lower set-point temperature increase. As can be seen, the dashed lines do not continue to increase, because the respective processes can be controlled by the PI controller. Up to the point where the dashed line becomes visible, both simulations follow the same trajectory. With these two simulations it can be detected when the system stability is actually lost. The loss of stability must occur between the point in time where each dashed line becomes visible and where the solid line reaches the set-point temperature. The point of loss of stability for all processes are indicated by dash-dotted lines parallel to the $\mathrm{y}$-axis of Figure 2 . For processes $\mathrm{P}_{1}^{1}, \mathrm{P}_{2}^{1}$, and $\mathrm{P}_{3}^{1}$ the loss of stability occurs at a time of $0.57 \mathrm{~h}, 0.71 \mathrm{~h}$ and $0.60 \mathrm{~h}$, respectively. The times when stability is lost for processes $\mathrm{P}_{4}^{1}, \mathrm{P}_{5}^{1}$, and $\mathrm{P}_{6}^{1}$ are given by $0.63 \mathrm{~h}, 0.68 \mathrm{~h}$, and $0.50 \mathrm{~h}$.

The next step of the verification of stability criterion $\mathcal{K}$ requires to compare the times when the system actually becomes unstable, when criterion $\mathcal{K}$ identifies the system to become unstable, and what the value of $\mathcal{K}$ is at the point in time when stability is lost. As was 


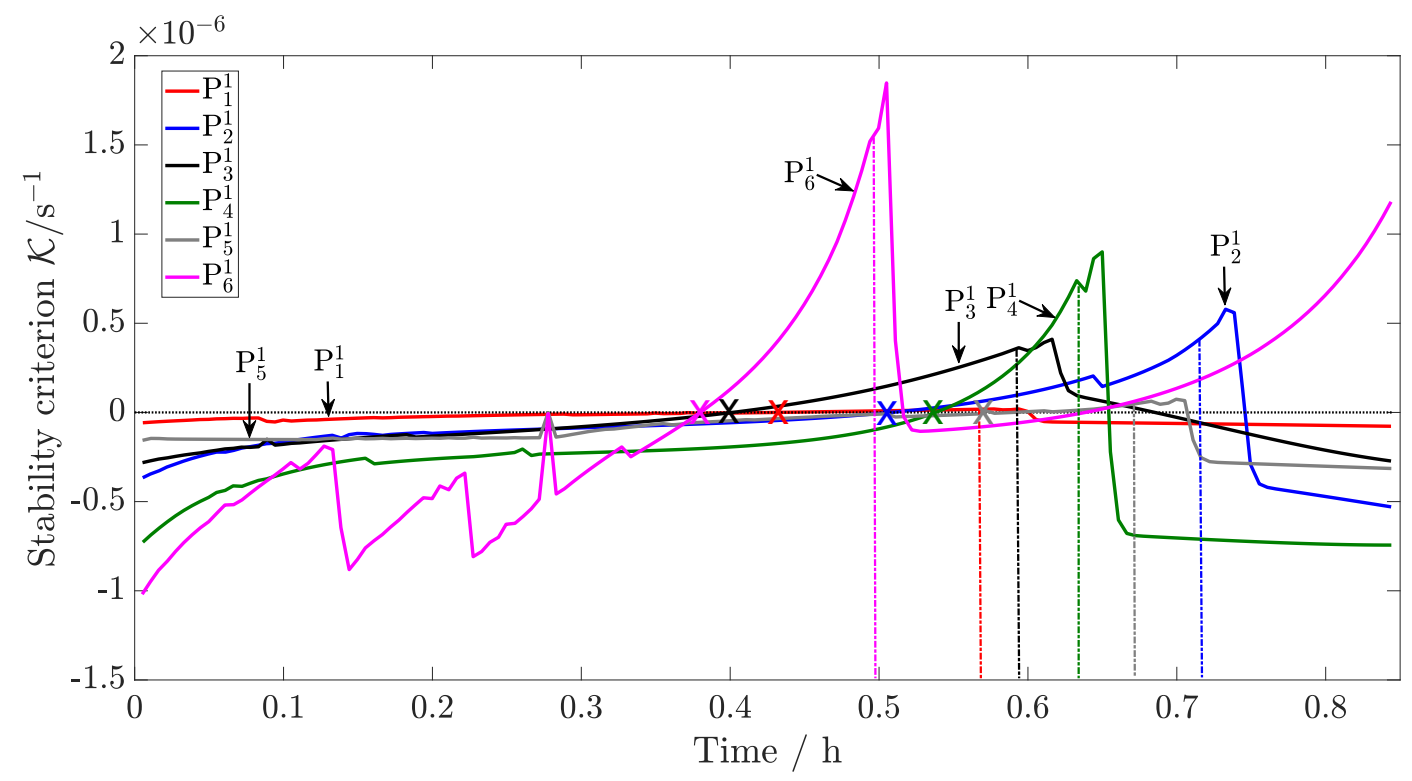

Figure 3: $\mathcal{K}$ criterion profiles for processes $\mathrm{P}_{1}^{1}-\mathrm{P}_{6}^{1}$. The dotted line within the figure indicates where $\mathcal{K}=0$. The crosses indicate where thermal stability criterion $\mathcal{K}$ detects an unstable process. The dash-dotted lines parallel to the $y$-axis indicate when stability is lost in the system.

indicated in Figure 2, the time at which stability is lost is indicated by dash-dotted lines parallel to the y-axis. The stability criterion profiles for processes $\mathrm{P}_{1}^{1}-\mathrm{P}_{6}^{1}$ are shown in Figure 3.

In Figure 3 it is seen that for each process the criterion gives a positive number at the point in time where stability is lost, indicated by the dash-dotted lines. This is in agreement with Kähm and Vassiliadis (2018d) and Kähm and Vassiliadis (2018c), where similar results were obtained for single reaction systems. Hence criterion $\mathcal{K}$ correctly indicates that an unstable process is present when the thermal stability of the system, as shown in Figure 2, is lost.

The crosses in Figure 3 indicate where each profile for $\mathcal{K}$ has a value of zero. This is the switch-over point which indicates a thermally unstable process is present. The first positive feature is that instability is predicted before it occurs. This can be observed in Figure 3 as the crosses occur before the dash-dotted lines indicating the loss of stability in the system. Furthermore, the difference in time between the real loss of stability and the prediction of the loss of stability are separated by approximately $0.2 \mathrm{~h}$, which is equivalent to 12 minutes. This should give enough time for an advanced control scheme to be able to modify the control actions to keep the system in a stable regime.

For each process given in Figure 3 the value of $\mathcal{K}$ reduces sharply once the system becomes unstable after approximately $0.05 \mathrm{~h}$. This is the case as the sharp increase in reaction temperature leads to a sharp increase in the rate of consumption of reagents. Criterion 
$\mathcal{K}$ incorporates the trajectories of both, temperatures and concentrations, which is why a sudden drop in concentrations will lead to a sudden change in the value of $\mathcal{K}$. The fact that the value becomes negative after the loss of stability has occurred is not in contradiction to the definition of $\mathcal{K}$. The purpose of thermal stability criterion $\mathcal{K}$ is to identify the point at which stability is lost, and not to predict how unstable a process is once stability is lost.

For processes $\mathrm{P}_{4}^{1}-\mathrm{P}_{6}^{1}$ in Figure 3 the values of $\mathcal{K}$ at the actual loss of stability are positive. Hence criterion $\mathcal{K}$ correctly classifies the point at which the loss of stability occurs. Furthermore, the crosses within Figure 3 show that criterion $\mathcal{K}$ predicts the stability to be lost at times occurring before the actual stability is lost. The difference in time between the predicted loss of stability, and the actual loss of stability, as indicated on the temperature profiles on Figure 2, is approximately $0.1 \mathrm{~h}$ for each process, hence giving 6 minutes for an advanced control scheme to react.

For process $\mathrm{P}_{6}^{1}$ it is further noted, that the profile of $\mathcal{K}$ at times before $0.4 \mathrm{~h}$, the time when instability is predicted, follows an oscillatory profile. As can be observed on Figure 2, the temperature profile at times before $0.4 \mathrm{~h}$ follows an oscillatory profile as well. This is the case because the PI controller acts very fast to cool the system once the initial set-point temperature of $389 \mathrm{~K}$ is reached. Since the PI controller was not tuned in order to give the best performance, this oscillatory effect is present for the temperature profile. The value of $\mathcal{K}$ is evaluated using information from the temperature and concentration trajectories of the system. Hence, sharp changes in the temperature will result in sharp changes in the value of $\mathcal{K}$. Therefore, the initial profile of $\mathcal{K}$ is given by the profile shown in Figure 3 . The other significant feature of the profile of $\mathcal{K}$ for process $\mathrm{P}_{6}^{1}$ is that the value of $\mathcal{K}$ increases after the loss of stability has occurred. This is different to processes $\mathrm{P}_{1}^{1}-\mathrm{P}_{5}^{1}$, where the value of $\mathcal{K}$ entered the negative region and decreased afterwards. In this case the reaction still has enough reagents to cause an accelerated rate of increase in temperature, which can be observed in Figure 2. As $\mathcal{K}$ follows the temperature and concentration profiles, in this process the effect of the temperature increasing at an accelerated rate outweighs the decrease in concentration, therefore increasing the value of $\mathcal{K}$.

Stability criterion $\mathcal{K}$ was shown to work for reaction scheme 1 . In the next section MPC is used with criterion $\mathcal{K}$ to intensify all batch processes, including the industrial reaction case studies given in Section 3.2.3.

\subsection{Verification of criterion $\mathcal{K}$ for reaction scheme 2}

A more complex reaction network is considered next, given by reaction scheme 2 . The reactions occurring in this reaction scheme are given in Section 3.2.2 with all data used. The equation of thermal stability criterion $\mathcal{K}$ used for this reaction scheme is similar to the expression in Equation (4.1) for reaction scheme 1. For reaction scheme 2 the effect of the 


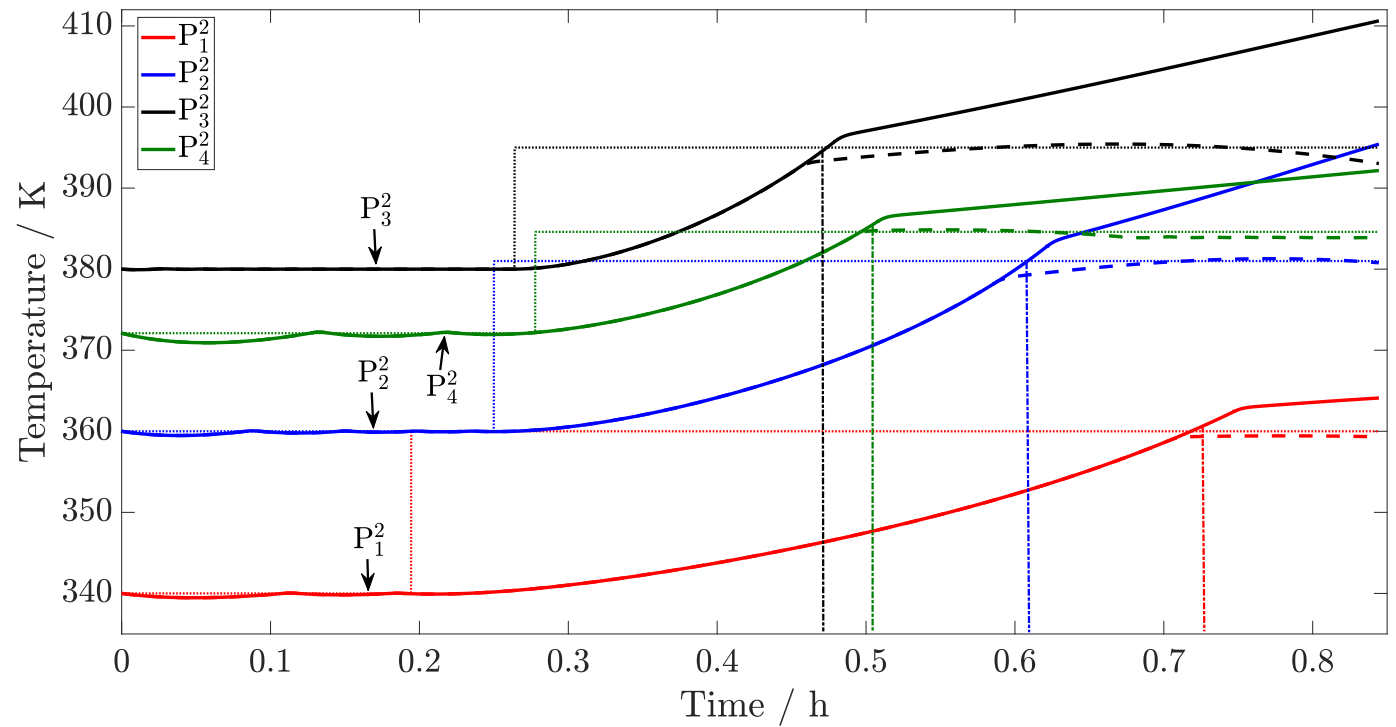

Figure 4: Temperature profiles for processes $\mathrm{P}_{1}^{2}-\mathrm{P}_{4}^{2}$. The dashed lines indicate the set-point temperatures for the PI controller. The dash-dotted lines parallel to the y-axis indicate when stability is lost in the system.

additional two reactions and the respective expression for the divergence of the Jacobian have to be added. The two components added to Equation (4.1) are given by:

$$
\begin{aligned}
& +\mathcal{D}_{5}^{(s-1)}\left[m_{B} \frac{B_{5}^{(s)}-B_{5}^{(s-1)}}{B_{5}^{(s-1)}}+m_{\gamma} \frac{\gamma_{5}^{(s)}-\gamma_{5}^{(s-1)}}{\gamma_{5}^{(s-1)}}+m_{\mathrm{Da}_{\mathrm{res}}} \frac{\mathrm{Da}_{\mathrm{res}, 5}^{(s)}-\mathrm{Da}_{\mathrm{res}, 5}^{(s-1)}}{\mathrm{Da}_{\mathrm{res}, 5}^{(s-1)}}\right] \\
& +\mathcal{D}_{6}^{(s-1)}\left[m_{B} \frac{B_{6}^{(s)}-B_{6}^{(s-1)}}{B_{6}^{(s-1)}}+m_{\gamma} \frac{\gamma_{6}^{(s)}-\gamma_{6}^{(s-1)}}{\gamma_{6}^{(s-1)}}+m_{\mathrm{Da}_{\mathrm{res}}} \frac{\mathrm{Da}_{\mathrm{res}, 6}^{(s)}-\mathrm{Da}_{\mathrm{res}, 6}^{(s-1)}}{\mathrm{Da}_{\mathrm{res}, 6}^{(s-1)}}\right]
\end{aligned}
$$

The dimensionless variables and the form of the generalized equation for criterion $\mathcal{K}$ are given in Section 2.3. The expressions for $\mathcal{D}_{1}^{(s-1)}-\mathcal{D}_{4}^{(s-1)}$ for reaction scheme 2 are the same as for reaction scheme 1. The expressions for $\mathcal{D}_{5}^{(s-1)}$ and $\mathcal{D}_{6}^{(s-1)}$ are calculated in the same manner as $\mathcal{D}_{1}^{(s-1)}$ in Equation (4.2).

The same analysis as for reaction scheme 1 is carried out for reaction scheme 2 . In order to prove that the same level of reliability is achieved as the reaction network increases in size, 6 simultaneous reactions as described in Section 3.2.2 are considered here. As was done for reaction scheme 1, two simulations are carried out per process: one simulation shows an initially stable system becoming unstable after an increase in set-point temperature. The second simulation of the same process involves a smaller increase in set-point temperature, resulting in a stable system after this set-point increase. The temperature profiles for processes $\mathrm{P}_{1}^{2}-\mathrm{P}_{4}^{2}$ are shown in Figure 4. 


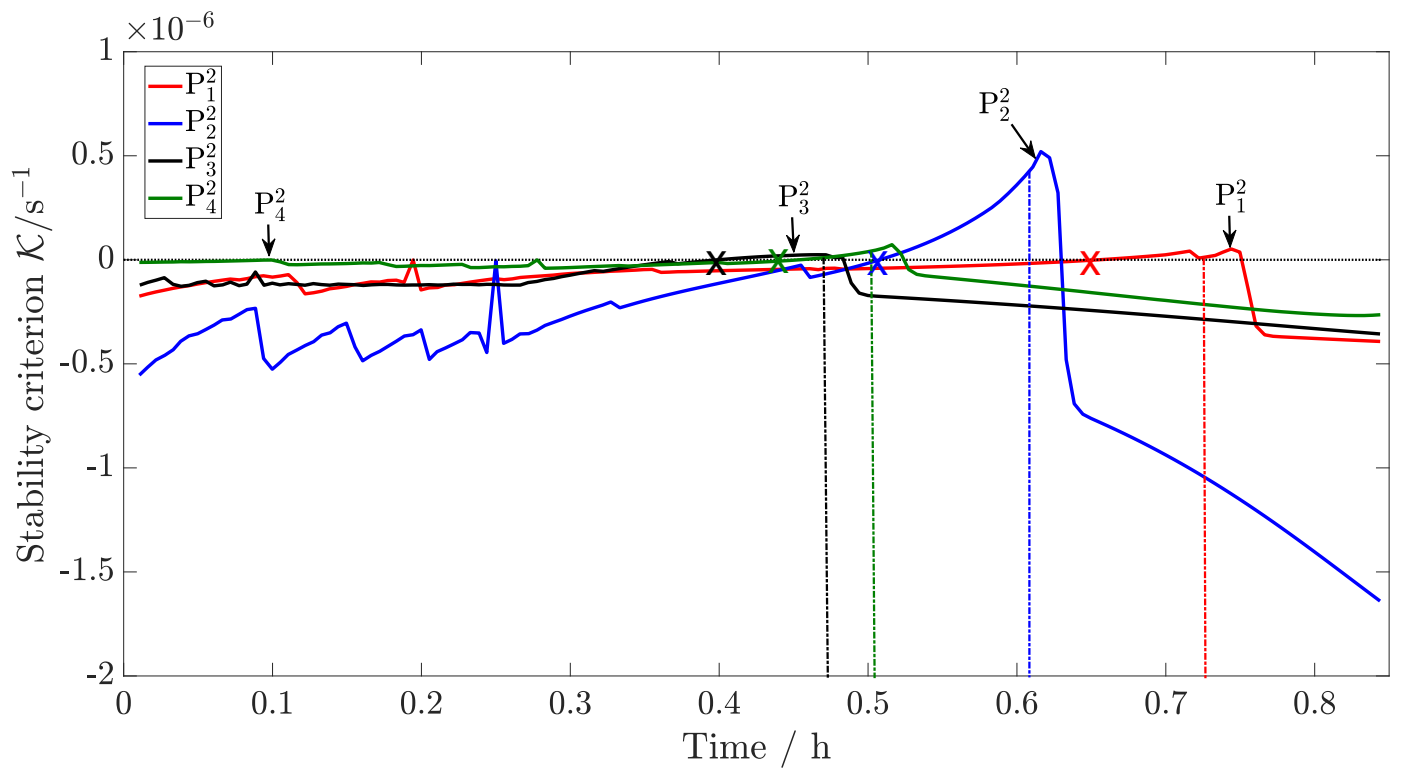

Figure 5: $\mathcal{K}$ criterion profiles for processes $\mathrm{P}_{1}^{2}-\mathrm{P}_{4}^{2}$. The dotted line indicates where $\mathcal{K}=0$. The crosses indicate where thermal stability criterion $\mathcal{K}$ detects an unstable process. The dash-dotted lines parallel to the $\mathrm{y}$-axis indicate when stability is lost in the system.

In Figure 4 it can be seen that the solid lines representing temperatures increase further than the set-point temperatures. This is the case because thermally unstable systems are obtained for the set-point temperature given by the dotted lines. The PI controller cannot cool the systems enough to avoid thermal runaway behavior, even when opening the cooling valve completely. The dashed lines representing the temperature profiles of the system with a smaller increase in set-point temperature show that stable systems can be achieved. Initially stable processes are present. As was done for the processes in reaction scheme 1, the point at which thermal stability is lost can be identified by comparing the stable and unstable systems, between which stability is lost. The point at which stability is lost is indicated by vertical dash-dotted lines given in Figure 4. These will be shown for the thermal stability criterion as well, in order to examine how well criterion $\mathcal{K}$ predicts the thermal instability.

The verification of $\mathcal{K}$ requires to check where the systems shown in Figure 4 become unstable, and where $\mathcal{K}$ predicts them to be unstable. Furthermore it is important to see what the value of $\mathcal{K}$ is at the point where stability is actually lost, indicated by dash-dotted lines. A plot of $\mathcal{K}$ for processes $\mathrm{P}_{1}^{2}-\mathrm{P}_{4}^{2}$ is shown in Figure 5.

Firstly it is noted from Figure 5 that, as for reaction scheme 1, the prediction of stability indicated by the crosses occurs before the systems lose stability, as indicated by the vertical dash-dotted lines. Secondly, at times where stability is lost, given by $0.73 \mathrm{~h}, 0.61 \mathrm{~h}, 0.48 \mathrm{~h}$ and $0.51 \mathrm{~h}$ for processes $\mathrm{P}_{1}^{2}-\mathrm{P}_{4}^{2}$, respectively, the values of $\mathcal{K}$ are all positive, hence classifying this as an unstable point. The difference in times between the prediction of instability 
(crosses) and the times of actual loss of stability (dash-dotted lines) is approximately 0.1 $\mathrm{h}$ for each process, hence giving a time of approximately 6 minutes to react. This is more than enough for advanced control schemes, and not too large to make the stability prediction too conservative. In case of plant-model mismatch this property is very useful, as predicting instability before it occurs is essential.

The time difference between actual loss of thermal stability and thermal stability prediction is obtained due to the nature of criterion $\mathcal{K}$ and how its gradient coefficients are found, as outlined in Kähm and Vassiliadis (2018c) and Kähm and Vassiliadis (2018d). The time difference, giving a safety margin, is therefore a feature of how criterion $\mathcal{K}$ is defined and it is not possible to design criterion $\mathcal{K}$ to result in a certain time difference between predicted and actual loss of thermal stability. A more detailed discussion on this can be found in Kähm and Vassiliadis $(2018 \mathrm{c}, \mathrm{d})$.

For process $\mathrm{P}_{2}^{2}$ the same oscillatory behavior as for process $\mathrm{P}_{6}^{1}$ can be observed. This is again due to the PI controller at the initial stable operating temperature: since the PI controller is not perfectly tuned, the cooling action cools down the system very quickly, causing oscillatory behavior in the temperature as can be seen in Figure 4. Since criterion $\mathcal{K}$ takes into account information from the temperature and concentration trajectories, the sudden changes in temperature on Figure 4 will cause similar effects on the trajectory of $\mathcal{K}$. This is exactly what is observed for process $\mathrm{P}_{2}^{2}$ in Figure 5.

Stability criterion $\mathcal{K}$ was shown to work for reaction schemes 1 and 2 . In the next section MPC is used with criterion $\mathcal{K}$ to intensify batch processes $\mathrm{P}_{1}^{1}-\mathrm{P}_{6}^{1}$, as well as the nitration of toluene presented in Section 3.2.3.

\section{Intensification of batch processes with MPC}

\subsection{MPC frameworks}

The intensification of batch processes enables the reduction of processing times, hence improving the efficiency of chemical processes. This can be achieved by continuously increasing the reaction temperature. Many batch processes in industry employ a constant set-point temperature policy for which the process is guaranteed to run in a stable regime. This is achieved by starting the process in a low enough temperature where the process is known to be controllable, and then this temperature is kept constant with PID control.

Model Predictive Control (MPC) is an advanced control scheme capable of dealing with system constraints. This algorithm solves an Optimal Control Problem (OCP) for every MPC step to find new control inputs (Rawlings and Mayne, 2015; Christofides et al., 2011).

The intensification of batch processes requires the full nonlinear model as there is no steady-state operating point. This condition presents issues with respect to defining stable 
operating points, which is why a different solution to this issue is required. For this reason a modified MPC framework is employed: the generalization of thermal stability criterion $\mathcal{K}$ is now embedded within an MPC framework according to Kähm and Vassiliadis (2018d,c). The optimization problem solved at every MPC step in this work is given by:

$$
\min _{u} \int_{t_{0}}^{t_{0}+t_{p}}\left(T_{\mathrm{R}}-T_{\mathrm{sp}}\right)^{2} \mathrm{~d} t
$$

subject to:

$$
\begin{aligned}
g(x, y, u, t) & =\dot{x} \\
h(x, y, u, t) & =0 \\
\mathcal{K}(t) & \leq 0 \\
X_{\mathrm{A}}\left(t_{\text {reac }}\right) & =70 \% \\
t_{0} \leq t^{(s)} & \leq t_{0}+t_{p}
\end{aligned}
$$

where $t_{\text {reac }}$ is the time required to reach the final conversion of the reaction, $x$ are the differential variables, $y$ are the algebraic variables, $u$ are the control variables, $\mathcal{K}(t)$ is the profile of $\mathcal{K}, t_{0}$ and $t_{0}+t_{p}$ are the initial and final times of the current MPC step, respectively, and $X_{\mathrm{A}}\left(t_{\mathrm{reac}}\right)$ is the conversion of component $\mathrm{A}$ at final time $t_{\mathrm{reac}}$. A more detailed description of the MPC structure employed in this work is given in Kähm and Vassiliadis (2018d,c).

The MPC formulation shown in Equation (5.1) is valid once it ensured the process is safe at initial time. If criterion $\mathcal{K}$ cannot be satisfied thermal runaway behavior is predicted and hence the appropriate shut down procedure has to be initiated. Stable operation can be achieved again by use of additional external cooling not used for regular operation, or by addition of inhibitors as commonly done for polymerization reactions.

To compare the performance of using an additional constraint, as given in Equation (5.1), three MPC frameworks are considered.

MPC framework 1 uses thermal stability criterion $\mathcal{K}$ as an additional constraint as shown in Equation (5.1d). The control horizon is set to $60 \mathrm{~s}$, with 6 control steps of $10 \mathrm{~s}$, while the prediction horizon is set to $80 \mathrm{~s}$. As will be shown, these time horizons with stability criterion $\mathcal{K}$ give stable control with small computational time.

MPC framework 2 uses a constant temperature set-point for which the processes are thermally stable. The control horizon is set to $30 \mathrm{~s}$, with 3 control steps of $10 \mathrm{~s}$, and a prediction horizon of $50 \mathrm{~s}$. It is not necessary for the prediction horizon to be large for this MPC framework, as no change in temperature set-point occurs during the processes. This 
also means, that an overall longer reaction time is expected in order to reach the target conversion for the reaction.

MPC framework 3 uses the standard nonlinear MPC structure with an extended control horizon of $100 \mathrm{~s}$, with 10 control steps of $10 \mathrm{~s}$, and a prediction horizon of $300 \mathrm{~s}$. This is to ensure that stable control is obtained as the set-point temperature is increased. The resulting process control and computational time required per iteration will be compared between each MPC framework.

In order to compare the performance of each MPC framework, each process starts at the same initial temperature. This temperature is chosen to be close to the point of instability, hence representing the highest possible temperature with which a constant set-point temperature process, as given by MPC framework 2, can be carried out. The results are compared in terms of thermal stability, time to reach final conversion or reagent $\mathrm{A}$, and the computational time required for each MPC framework. For industry it is essential that the control system leads to a thermally stable process with a short reaction duration and small computational time.

The extent of intensification is compared by considering the conversion profiles for reagent A, given by $X_{\mathrm{A}}$ :

$$
X_{\mathrm{A}}=\frac{[\mathrm{A}]_{0}-[\mathrm{A}]}{[\mathrm{A}]_{0}} \times 100 \%
$$

where $[\mathrm{A}]_{0}$ and $[\mathrm{A}]$ are the initial and current concentrations of reagent $\mathrm{A}$, respectively. The target conversion is set to $X_{\mathrm{A} \text {,target }}=70 \%$. Hence the faster this target conversion is reached without causing thermal runaway behavior, the more the process is intensified successfully.

Finally, MPC framework 1 is applied to the industrial case study (Halder et al., 2008) presented in Section 3.2.3. The results are compared to the solutions obtained in Kähm and Vassiliadis (2018b), where a similar MPC framework with a different thermal stability criterion, given by Lyapunov exponents (Strozzi and Zaldívar, 1994), was employed. The same industrial process is simulated in both cases, enabling the comparison of computational time for both MPC frameworks. Embedding Lyapunov exponents in an MPC framework requires a detailed analysis of the time horizon and initial perturbation, defining Lyapunov exponents.

The objective function in Equation (5.1a) penalizes deviations from the set-point temperature throughout the time frame of the optimal control step. In the simulations shown below the set-point temperature for MPC frameworks 1 and 3 are set to the maximum allowable temperature of $T_{\text {chem }}=470 \mathrm{~K}$. For MPC framework 2 , the initial temperature is set as the set-point temperature. 


\subsection{Intensification of batch processes with reaction scheme 1}

The intensification of batch processes is first considered for reaction scheme 1 . In this reaction scheme 4 simultaneous reactions occur according to Section 3.2.1. Given each MPC framework, the resulting temperature and conversion profiles are examined. Important for the application to industrial processes is the time to reach the target conversion of $X_{\mathrm{A}, \text { target }}=70 \%$. Furthermore it is essential that each process is kept under control, never exceeding the maximum allowable temperature of $T_{\text {chem }}=470 \mathrm{~K}$. Finally, the computational time required for every MPC iteration for each MPC framework is compared. The smaller the computational time, the more feasible its application to industry. Furthermore, it is important that the computational time is below the time available given by the control horizon.

For clarity only Figures for processes $\mathrm{P}_{5}^{1}$ and $\mathrm{P}_{6}^{1}$ are shown below for reaction scheme 1 . Similar solutions to those obtained for processes $\mathrm{P}_{5}^{1}$ and $\mathrm{P}_{6}^{1}$ are obtained for all remaining processes of reaction scheme 1 . The temperature profiles when each MPC framework is applied to these processes are shown in Figure 6a.

MPC framework 1 , embedding criterion $\mathcal{K}$, shows a continuous increase in system temperature, without exceeding the maximum allowable temperature $T_{\text {chem }}$. For processes $\mathrm{P}_{5}^{1}$ and $\mathrm{P}_{6}^{1}$ stable reactions are obtained. The initial temperatures for these processes are equal to the one given for MPC framework 3. This continuous increase in temperature will result in a more efficient process when compared to MPC framework 2. This will be shown in the conversion profiles below. Furthermore, the upper limit of the temperature, $T_{\text {chem }}$, is not exceeded.

MPC framework 2 results in constant temperature throughout each process. No thermal runaway occurs for processes $\mathrm{P}_{5}^{1}$ and $\mathrm{P}_{6}^{1}$, as the temperature is kept constant during the process. The trade-off of having an overly conservative process run at a constant temperature is outlined when considering the conversion profiles for each process.

MPC framework 3 results in thermal runaway behavior even with an extended control and prediction horizon. The temperature increases in an uncontrolled manner, exceeding the maximum allowable temperature. The maximum temperatures of $910 \mathrm{~K}$ and $1200 \mathrm{~K}$ are reached at times of $0.9 \mathrm{~h}$ and $1.5 \mathrm{~h}$ for processes $\mathrm{P}_{5}^{1}$ and $\mathrm{P}_{6}^{1}$, respectively. At these peak temperatures an explosion would occur in real processes if no preventative actions were to be taken.

To examine further how well each process is intensified, the conversion profiles for each MPC framework are considered next. The time required to reach the target conversion of $70 \%$ is found and compared. The smaller the time required, the more the process is intensified. As was shown for the temperature profiles, it is expected that the processes controlled by MPC framework 3 are intensified most whilst keeping the process under control. 


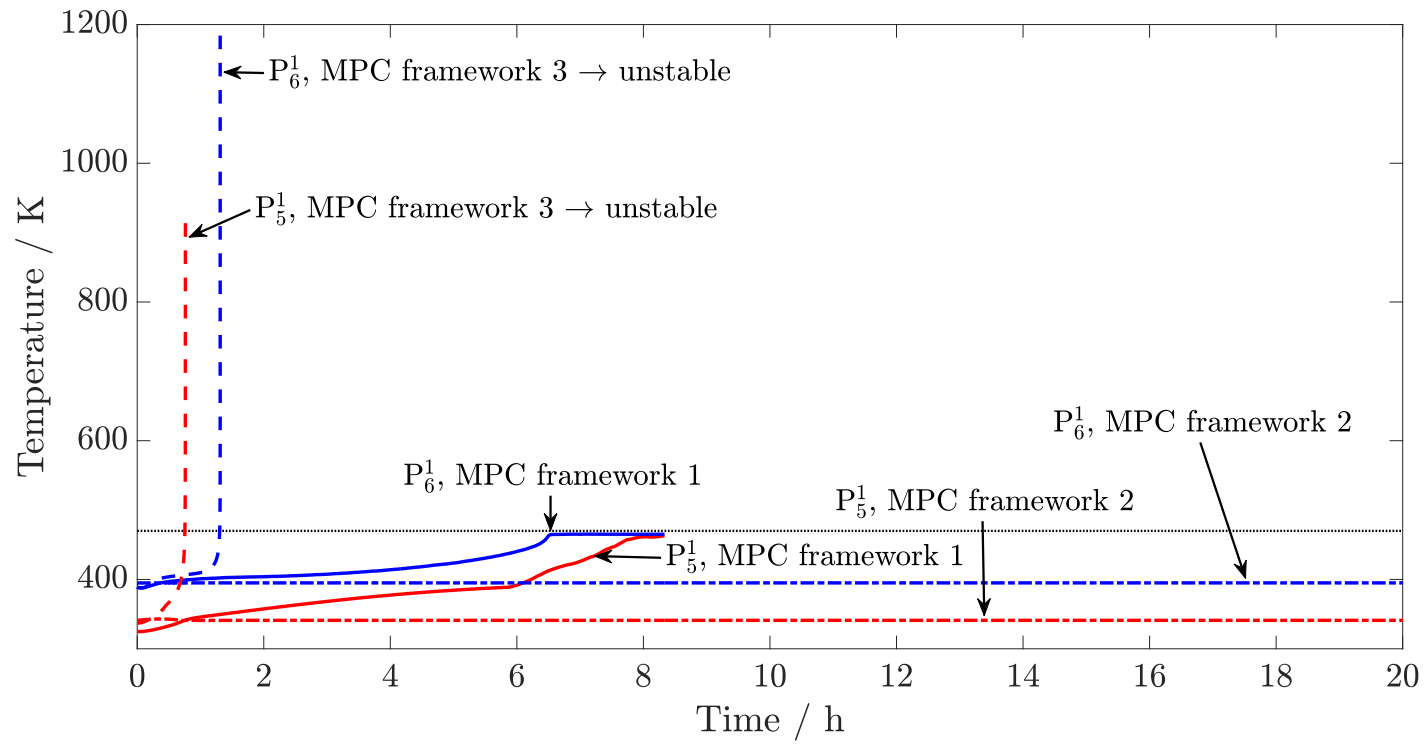

(a) Temperature profiles for processes $\mathrm{P}_{5}^{1}-\mathrm{P}_{6}^{1}$ with all three MPC frameworks. The solid, dash-dotted and dashed lines show the temperature profiles for MPC frameworks 1, 2 and 3, respectively. The dotted line indicates the maximum allowable temperature of $T_{\text {chem }}=470 \mathrm{~K}$.

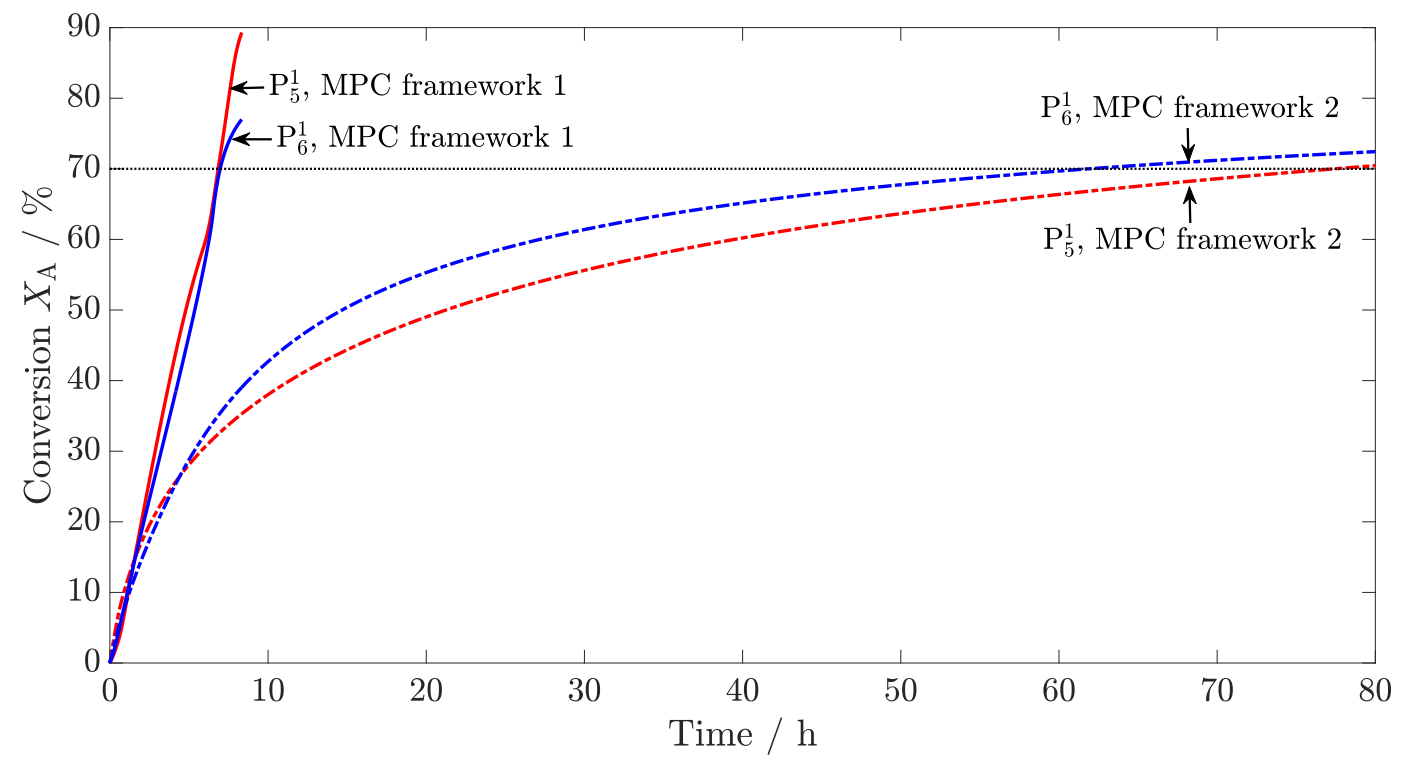

(b) Conversion profiles of reagent A for processes $\mathrm{P}_{5}^{1}$ and $\mathrm{P}_{6}^{1}$ controlled by MPC frameworks 1 and 2 . The solid and dash-dotted lines show the conversion profiles for MPC frameworks 1 and 2, respectively. The dotted line indicates the target conversion of $X_{\mathrm{A}, \text { target }}=70 \%$.

Figure 6: Results for the intensification of processes $\mathrm{P}_{5}^{1}$ and $\mathrm{P}_{6}^{1}$ with MPC frameworks 1,2 and 3. 
Table 5: Summary of results obtained fro reaction scheme 1 controlled by each of the three MPC frameworks presented, where $t_{\text {reac }}$ is the time required to each the target conversion of $X_{\mathrm{A} \text {,target }}=70 \%, T_{\text {peak }}$ is the peak temperature reached during the process, which is not allowed to exceed $470 \mathrm{~K}$, and $\bar{t}_{\text {comp }}$ is the average computational time required to evaluate each MPC step.

\begin{tabular}{ccccccccc}
\hline & \multicolumn{3}{c}{ MPC framework 1 } & \multicolumn{3}{c}{ MPC framework 2} & \multicolumn{3}{c}{ MPC framework 3 } \\
& $t_{\text {reac }} / \mathrm{h}$ & $T_{\text {peak }} / \mathrm{K}$ & $\bar{t}_{\text {comp }} / \mathrm{CPUs}$ & $t_{\text {reac }} / \mathrm{h}$ & $T_{\text {peak }} / \mathrm{K}$ & $\bar{t}_{\text {comp }} / \mathrm{CPUs}$ & $T_{\text {peak }} / \mathrm{K}$ & $\bar{t}_{\text {comp }} / \mathrm{CPUs}$ \\
\hline $\mathrm{P}_{1}^{1}$ & 5.2 & 467 & 0.51 & $>150$ & 355 & 0.11 & 705 & 2.2 \\
$\mathrm{P}_{2}^{1}$ & 2.7 & 469 & 0.82 & 6.1 & 368 & 0.50 & 510 & 2.5 \\
$\mathrm{P}_{3}^{1}$ & 2.7 & 469 & 0.94 & 16.7 & 383 & 0.97 & 485 & 3.3 \\
$\mathrm{P}_{4}^{1}$ & 5.1 & 468 & 0.58 & 147 & 399 & 0.62 & 921 & 3.5 \\
$\mathrm{P}_{5}^{1}$ & 7.0 & 467 & 0.67 & 78 & 344 & 0.57 & 923 & 3.1 \\
$\mathrm{P}_{6}^{1}$ & 7.0 & 469 & 0.59 & 61 & 401 & 0.41 & 1204 & 3.8 \\
\hline
\end{tabular}

For clarity only the conversion of processes $\mathrm{P}_{5}^{1}$ and $\mathrm{P}_{6}^{1}$ are considered, as these can be compared to the temperature profiles given in Figure 6a. The profiles for the conversion of reagent $\mathrm{A}$ for processes $\mathrm{P}_{5}^{1}$ and $\mathrm{P}_{6}^{1}$ are shown in Figure $6 \mathrm{~b}$.

MPC framework 1 results in stable control, as was shown in Figure 6a, whilst increasing the reaction temperature continuously. The target conversion of $70 \%$ is reached after $7.0 \mathrm{~h}$ and $7.1 \mathrm{~h}$ for processes $\mathrm{P}_{5}^{1}$ and $\mathrm{P}_{6}^{1}$, respectively. This is a significant decrease in reaction time with respect to MPC framework 2, while also keeping the process under control at every point in time.

Stable control is achieved with MPC framework 2, at the expense of long reaction times: the target conversion is reached after $78 \mathrm{~h}$ and $61 \mathrm{~h}$ for processes $\mathrm{P}_{5}^{1}$ and $\mathrm{P}_{6}^{1}$, respectively. Having a constant reaction temperature hence has advantages in terms of reactor stability, and disadvantages in terms of efficiency.

With processes $\mathrm{P}_{1}^{1}-\mathrm{P}_{6}^{1}$ controlled by MPC frameworks 1, 2 and 3 it is shown that MPC framework 1 results in stable control and intensified processes to increase process efficiency. The decrease in reaction time when compared to MPC framework 2 is at least 3-fold. MPC framework 3 shows unstable behavior, causing thermal runaways. This is the case although a larger control and prediction horizon than for the other MPC frameworks is used.

The last important feature of all these MPC frameworks to note is the computational time required to use each of these MPC frameworks. The smaller the computational time, the more feasible the application to industrial processes. The average computational times per MPC step, $\bar{t}_{\text {comp }}$, together with the time to reach the target conversion, $t_{\text {reac }}$, and the peak temperature throughout each process, $T_{\text {peak }}$, are summarized in Table 5.

From Table 5 can be seen that MPC framework 3 results in peak temperatures $T_{\text {peak }}>$ $T_{\text {chem }}$, hence giving unfeasible processes. As shown in Figure 6a, the temperature profiles 
rise sharply due to thermal runaway behavior. Furthermore, MPC framework 3 requires the largest computational time per MPC step. This is the case because this MPC framework has the longest control and prediction horizon. Important to note is that this MPC framework is not able to keep the processes under control. A longer prediction horizon would be required to achieve stable control, leading to even larger computational times. Since the computational time is already close to or larger than $10 \mathrm{~s}$, this poses a problem for potential industrial applications.

MPC framework 2 gives close to constant temperature profiles as shown in Figure 6a. The initial temperatures are very close to the maximum temperatures $T_{\text {peak }}$. The time to reach the final conversion of $70 \%$ varies strongly from $6.1 \mathrm{~h}$ for process $\mathrm{P}_{2}^{1}$ to more than $150 \mathrm{~h}$ for process $\mathrm{P}_{1}^{1}$. This sets the baseline relative to which the intensification of MPC framework 1 is compared to. The initial temperatures for each process controlled with MPC framework 2 is close to the boundary of stability initially: a further increase in the initial temperature of $1 \mathrm{~K}$ would results in an unstable process. The computational time given in Table 5 for MPC framework 2 is the smallest amongst all MPC frameworks which is expected: a smaller control horizon with the objective of just keeping a constant temperature is much simpler than for the other MPC frameworks.

MPC framework 1 , embedding criterion $\mathcal{K}$, results in peak temperatures below the maximum allowable temperature $T_{\text {chem }}$. As is seen in Figure 6 a the temperature is increased in a controlled manner throughout the process, hence resulting in an intensified reaction. From the times required to reach the target conversion, $t_{\text {reac }}$, given in Table 5 it is seen that a speed-up of at least 3-fold is achieved when implementing MPC framework 1, compared to MPC framework 2. A controlled intensification results in much shorter reaction times, increasing the efficiency of the respective batch reactors. The computational times $\bar{t}_{\text {comp }}$ shown are larger than those for MPC framework 2, but less than half of those for MPC framework 3. To achieve stable control with MPC framework 3 even larger control and prediction horizons are necessary which increases the computational time even further. Hence, MPC framework 1 results in an efficient control system in terms of computational and economical cost.

\subsection{Intensification of batch process for the nitration of toluene}

The nitration of toluene presents a challenging case study of an exothermic reaction network of industrial interest. The parameters defining this reaction network are given in Sections 3.2.3 and 3.3. The goal of this case study is to show that criterion $\mathcal{K}$ can be applied successfully to an industrially relevant reaction and give similar results in terms of intensification, as for reaction scheme 1.

This case study was considered in Kähm and Vassiliadis (2018b) with a different method to evaluate the system stability, given by Lyapunov exponents. The reactor parameters used 
in Kähm and Vassiliadis (2018b) are used in this work for the results to be comparable. The reactor parameters used for the nitration of toluene are given in Table 1.

The intensification of the nitration of toluene is carried out with MPC framework 1 by starting the same reaction at three different initial temperatures. For this case study the maximum allowable temperature is set to $T_{\text {chem }}=510 \mathrm{~K}$. The objective function is formulated such that the most efficient process is found:

$$
\min _{u(t)} \Phi(x(t), y(t), u(t))=-\left[\mathrm{o}-\mathrm{C}_{7} \mathrm{H}_{7} \mathrm{NO}_{2}\right]\left(t_{f}\right)
$$

where $\left[\mathrm{o}-\mathrm{C}_{7} \mathrm{H}_{7} \mathrm{NO}_{2}\right]\left(t_{f}\right)$ is the concentration of the product at final time, $t_{f}$, hereafter referred to as o-nitrotoluene. The final concentration of o-nitrotoluene at each step $(s)$ of the MPC algorithm is optimized, resulting in the smallest possible time of reaction. The target concentration of o-nitrotoluene is set to $\left[\mathrm{o}-\mathrm{C}_{7} \mathrm{H}_{7} \mathrm{NO}_{2}\right]\left(t_{f}\right)=2.5 \mathrm{kmol} \mathrm{m}^{-3}$.

The application of MPC framework 1 uses a control horizon of $t_{c}=40 \mathrm{~s}$, with steps of length $10 \mathrm{~s}$, and a prediction horizon of $t_{p}=60 \mathrm{~s}$. The time required to find the control values set has to be shorter than the length of the first control value implemented. In this work this upper limit in computational time is given by $10 \mathrm{~s}$. Three different starting temperatures of $430 \mathrm{~K}, 440 \mathrm{~K}$, and $450 \mathrm{~K}$ are used to show that MPC framework 1 results in stable control for each of these cases. The temperature profiles for each process are shown in Figure 7a.

In Figure 7a no unstable process is obtained for any of the three processes. The maximum allowable temperature $T_{\text {chem }}=510 \mathrm{~K}$ is not exceeded for each process, hence each process is successfully intensified while satisfying the stability constraint given by thermal stability criterion $\mathcal{K}$.

The time required until the target concentration of o-nitrotoluene shows how well the intensification with MPC framework 1 performs for batch processes. The concentration profiles for each product obtained during the process are shown for each starting temperature in Figure 7b.

The concentration for o-nitrotoluene, given by the solid lines in Figure 7b, reaches the target concentration of $2.5 \mathrm{kmol} \mathrm{m}^{-3}$ within 7 hours, which is similar to the results obtained in Kähm and Vassiliadis (2018b), where Lyapunov exponents were used instead of criterion $\mathcal{K}$. Furthermore, the ratio of each nitrotoluene product obtained from the three different initial temperatures is equal in each case, as shown by the vertical dotted lines in Figure $7 \mathrm{~b}$. In Kähm and Vassiliadis (2018b) the computational time required for each starting temperature was approximately $9 \mathrm{~s}$ which is very close to the upper limit of the permissible computational time. The computational times required per MPC are $1.21 \mathrm{~s}, 1.75 \mathrm{~s}$, and $1.43 \mathrm{~s}$ for initial temperatures of $430 \mathrm{~K}, 440 \mathrm{~K}$, and $450 \mathrm{~K}$, respectively. If using Lyapunov exponents, the computational times required per MPC step when using criterion $\mathcal{K}$ are $8.9 \mathrm{~s}, 8.5 \mathrm{~s}$, and 9.1 


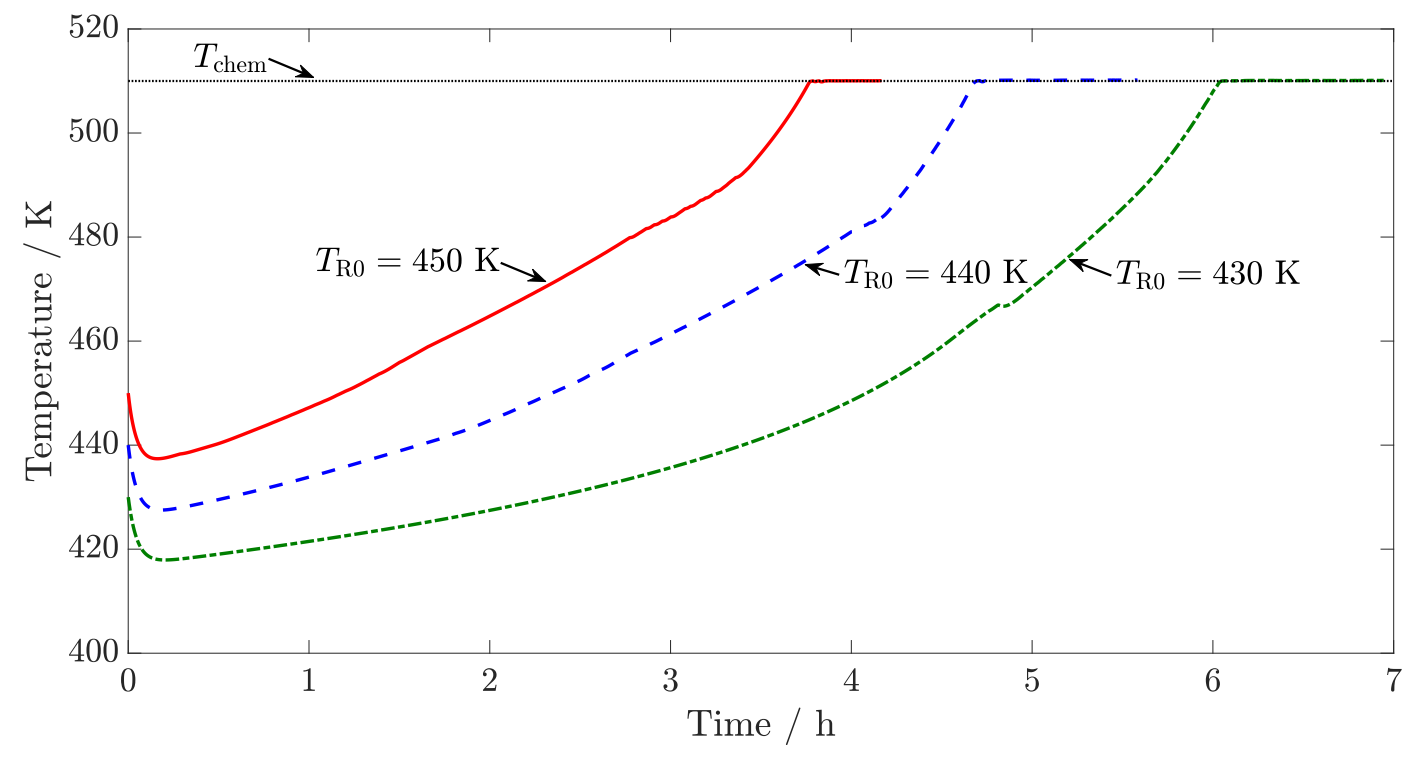

(a) Temperature profiles for intensified processes of the nitration of toluene. The solid line relates to $T_{\mathrm{R} 0}=450$ $\mathrm{K}$, the dashed line relates to $T_{\mathrm{R} 0}=440 \mathrm{~K}$ and the dash-dotted line relates to $T_{\mathrm{R} 0}=430 \mathrm{~K}$. The dotted line indicates the maximum allowable temperature of $T_{\text {chem }}=510 \mathrm{~K}$.

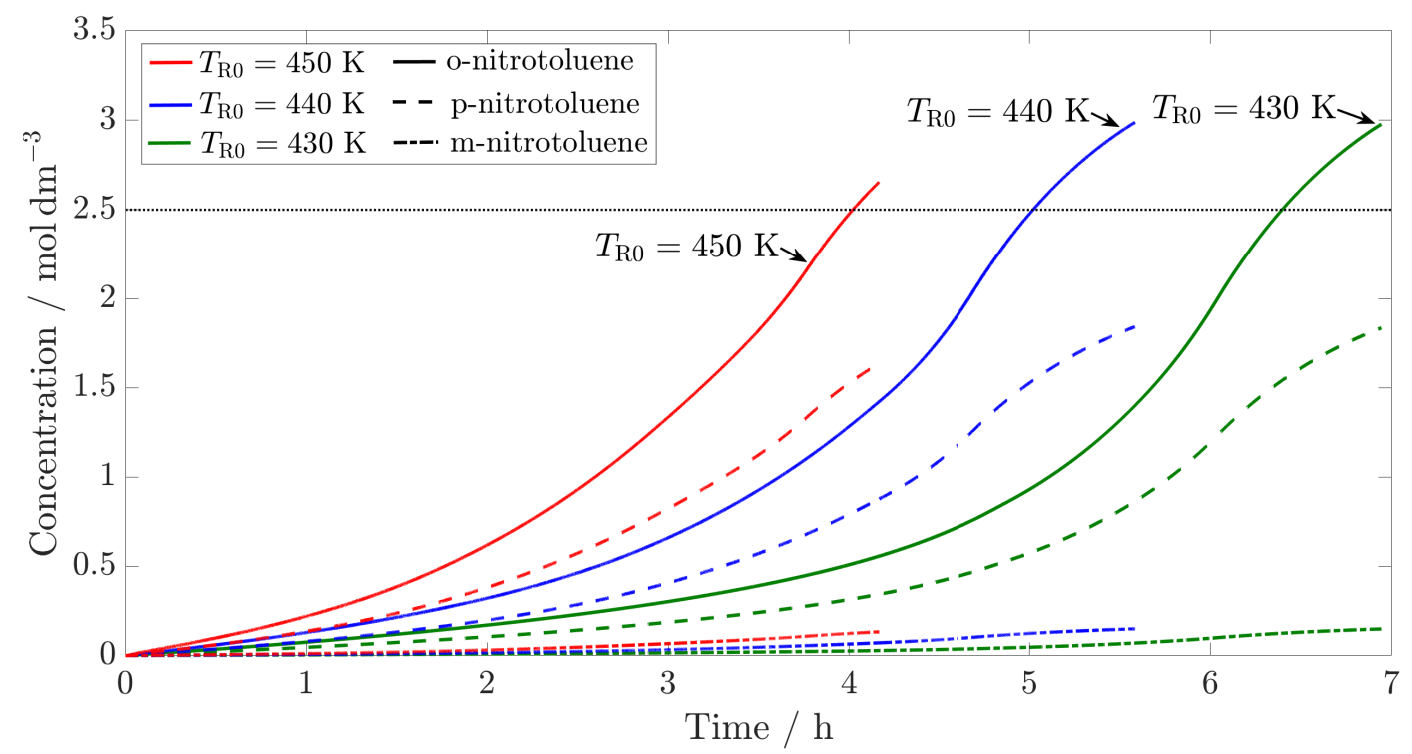

(b) Concentration profiles for the nitration of toluene reaction system. The profiles are obtained by control with MPC framework 1. The dotted line indicates the target concentration for o-nitrotoluene.

Figure 7: Results for the intensification of the nitration of toluene. 
s for initial temperatures of $430 \mathrm{~K}, 440 \mathrm{~K}$, and $450 \mathrm{~K}$, respectively (Kähm and Vassiliadis, 2018b). Hence it is seen that the computational time required with MPC framework 1, as presented in this work, is reduced by at least 4-fold compared to the framework using Lyapunov exponents as the measure of thermal stability (Kähm and Vassiliadis, 2018b). This shows that the same extent of intensification can be achieved with a more efficient MPC framework, whilst keeping the system under control at all times.

This last case study shows how the generalized expression for thermal stability criterion $\mathcal{K}$ can be implemented within a standard MPC framework, allowing a continuous increase in reactor temperature whilst keeping the respective batch process under control. This framework is valid for industrially relevant reactions, as is shown above. The computational time required is significantly shorter than for frameworks with Lyapunov exponents, hence resulting in an efficient and safe control scheme for highly exothermic batch processes.

\section{Conclusions and further work}

The thermal stability criterion $\mathcal{K}$, which was initially derived for single reaction systems (Kähm and Vassiliadis, 2018d,c), is successfully generalized to general reaction schemes in this work. It is shown that the instability of more complex reaction networks is reliably predicted using the generalized form of criterion $\mathcal{K}$. The thermal stability criterion predicts the instability approximately 10 minutes before it occurs in the real process. This is a positive feature of thermal stability criterion $\mathcal{K}$, because the prediction happens early enough so that action by the controllers can be taken to avoid thermal runaway behavior. The prediction of stability does not happen too early on the other hand, which would make it infeasible to intensify batch processes, as was shown for the divergence criterion in Kähm and Vassiliadis (2018d).

Nonlinear MPC is introduced and the main features of this advanced control scheme are shown. In this work four different nonlinear MPC frameworks were examined in terms of efficiency of the process, stability of the control, and the computational time required for the evaluation of each MPC framework. These three factors give rise to the feasibility of applying such an MPC framework in industry. It is further shown that criterion $\mathcal{K}$ can be applied to MPC frameworks as was done in Kähm and Vassiliadis (2018d) and Kähm and Vassiliadis (2018c). Embedding the thermal stability as an additional constraint within the MPC algorithm results in stable control, whilst increasing the reaction temperature continuously during the process. This results in much shorter reaction times when compared to MPC frameworks which keep a constant set-point temperature, as is often used in industry. This reduction in reaction time was shown to be at least 3-fold for the processes studied in this work. 
Comparing the performance of MPC embedded with stability criterion $\mathcal{K}$ and with Lyapunov exponents, it is shown that MPC with Lyapunov exponents results in larger computational time required. Both MPC frameworks give rise to stable control, but the framework using criterion $\mathcal{K}$ results in a more efficient control system. Furthermore, the computational time required to evaluate criterion $\mathcal{K}$ does not increase with the number of chemical reagents, as opposed to Lyapunov exponents.

Standard MPC frameworks with a larger control and prediction horizon are tested and result in unstable control. Thermal runaways were caused because the MPC framework did not recognize that the system entered an unstable operating regime. Furthermore, the computational time required by such an MPC framework with extended horizons is more than 3 times larger than for the MPC framework embedding stability criterion $\mathcal{K}$.

Since the values of $\mathcal{K}$ are usually of order $10^{-7}$ when close to the boundary of stability, the effect of parametric uncertainty and noisy measurements has to be considered further for potential industrial application. Model-plant mismatch in the system models is a further issue that will be considered in future work. Robust stability detection is of utmost importance for implementation of the proposed MPC framework in industry. The issue of parametric is currently being investigated using scenario based and worst case approaches for each relevant parameter in the batch reactor system. Whilst it is found that including parametric uncertainty results in more conservative process control, significant process intensification is still achieved. Measurement noise represents another issue to be addressed before successful implementation of this work in industry, because thermal stability prediction using criterion $\mathcal{K}$ relies heavily on trajectory information of all state variables. The authors therefore suggest the use of state estimation and filtering techniques, e.g. Kalman filters (Grewal and Andrews, 2015) and low-pass filters (Sedra and Smith, 2004), to ensure reliable information is used to evaluate criterion $\mathcal{K}$.

The MPC algorithm can be improved further if additional information for the optimizer can be obtained. Sensitivity or adjoint equations, if available, can be supplied to the optimizer to reduce the risk of numerical errors and instabilities, which can occur due to the finite differences scheme currently employed.

\section{Acknowledgments}

We thank the Engineering and Physical Sciences Research Council (EPSRC) and the Department of Chemical Engineering and Biotechnology, University of Cambridge, for funding the EPSRC PhD studentship for this project (DTP - University of Cambridge, Funder reference $\mathrm{EP} / \mathrm{M} 508007 / 1)$. 


\section{References}

Albalawi, F., Durand, H., Christofides, P.D., 2018. Process operational safety via model predictive control: Recent results and future research directions. Computers \& Chemical Engineering 114, 171-190.

Anagnost, J.J., Desoer, C.A., 1991. An elementary proof of the Routh-Hurwitz stability criterion. Circuits Systems Signal Process 10.

Bosch, J., Strozzi, F., Zbilut, J., Zaldívar, J.M., 2004. On-line runaway detection in isoperibolic batch and semibatch reactors using the divergence criterion. Computers and Chemical Engineering 28, 527-544.

Chen, L.P., Chen, W.H., Liu, Y., Peng, J.H., Liu, R.H., 2008. Toluene mono-nitration in a semi-batch reactor. Central European Journal of Energetic Materials 5, 37-47.

Christofides, P.D., Liu, J., Muñoz de la Peña, D., 2011. Networked and Distributed Predictive Control. Springer, London. chapter 2. pp. 13-45.

Chuong La, H., Potschka, A., Bock, H.G., 2017. Partial stability for nonlinear model predictive control. Automatica 78, 14-19.

Davis, M., Davis, R., 2003. Fundamentals of Chemical Reaction Engineering. McGraw-Hill. chapter 2. pp. 53-56.

Durand, H., Christofides, P.D., 2016. Actuator stiction compensation via model predictive control for nonlinear processes. American Institute of Chemical Engineers Journal 62, 2004-2023.

Ellis, M., Christofides, P.D., 2015. Real-time economic model predictive control of nonlinear process systems. American Institute of Chemical Engineers Journal 61, 555-571.

Green, D.W., Perry, R.H., 2008. Perry's Chemical Engineers' Handbook. eighth ed.. The McGraw-Hill. chapter 2.

Grewal, M.S., Andrews, A.P., 2015. Kalman filtering. John Wiley \& Sons, Inc.. chapter 8.

Halder, R., Lawal, A., Damavarapu, R., 2008. Nitration of toluene in a microreactor. Catalysis Today 125, 74-80.

Hirschfelder, J.O., Curtiss, C.F., Bird, R.B., 1955. Molecular theory of gases and liquids. American Insitute of Chemical Engieers Journal 1, 272. 
Huang, R., Biegler, L.T., Harinath, E., 2012. Robust stability of economically oriented infinite horizon NMPC that include cyclic processes. Journal of Process Control 22, 51-59.

Kähm, W., Vassiliadis, V.S., 2018a. Lyapunov exponents with model predictive control for exothermic batch reactors, in: IFAC-PapersOnLine.

Kähm, W., Vassiliadis, V.S., 2018b. Optimal laypunov exponent parameters for stability analysis of batch reactors with model predictive control. Computers and Chemical Engineering 119, 270-292.

Kähm, W., Vassiliadis, V.S., 2018c. Stability criterion for the intensification of batch processes with model predictive control. Chemical Engineering Research and Design 138, $292-313$.

Kähm, W., Vassiliadis, V.S., 2018d. Thermal stability criterion integrated in model predictive control for batch reactors. Chemical Engineering Science 188, 192-207.

Luo, K.M., Chang, J.G., 1998. The stability of toluene mononitration in reaction calorimeter reactor. Journal of Loss Prevention in the Process Industries 11, 81-87.

Mawardi, M., 1982. The nitration of monoalkyl benzene and the separation of its isomers by gas chromatography. Pertanika 5, 7-11.

Nocedal, J., Wright, S., 2006. Numerical Optimization. Springer. chapter 18. pp. 526-572.

Paul, E., Atiemo-Obeng, V., Kresta, S., 2004. Handbook of industrial mixing : science and practice. Wiley-Interscience.

Rawlings, J., Mayne, D., 2015. Model Predictive Control: Theory and Design. Nob Hill Publishing. chapter 1. pp. 1-60.

Rossi, F., Copelli, S., Colombo, A., Pirola, C., Manenti, F., 2015. Online model-based optimization and control for the combined optimal operation and runaway prediction and prevention in (fed-)batch systems. Chemical Engineering Science 138, 760-771.

Santos, L.O., de Oliveira, N.M., Biegler, L.T., 1995. Reliable and efficient optimization strategies for nonlienar model predictive control. Dynamics and Control of Chemical Reactors, Distillation Columns and Batch Processes (Dycord'95) , 33-38.

Sedra, A.S., Smith, K.C., 2004. Microelectronic circuits. Oxford University Press. chapter 12. Semenov, N., 1940. Thermal theory of combustion and explosion, in: Progress of Physical Science (U.S.S.R). 
Shampine, L., Reichelt, M., Kierzenka, J., 1999. Solving index-1 daes in matlab and simulink. SIAM Review 41, 538-552.

Sheats, G., Strachan, A., 1978. Rates and activation energies of nitronium ion formation and reaction in the nitration of toluene in $78 \%$ sulphuric acid. Canadian Journal of Chemistry 56, 1280-1283.

Sinnot, R., 2005. Chemical Engineering Design. Elsevier Butterworth-Heinemann. volume 6. chapter 12. pp. 634-638.

Strozzi, F., Zaldívar, J., 1994. A general method for assessing the thermal stability of batch chemical reactors by sensitivity calculation based on Lyapunov exponents. Chemical Engineering Science 49, 2681-2688.

Strozzi, F., Zaldívar, J., 1999. On-line runaway detection in batch reactors using chaos theory techniques. American Institute of Chemical Engineers Journal 45, 2429-2443.

Theis, A., 2014. Case study: T2 Laboratories explosion. Journal of Loss Prevention in the Process Industries 30, 296-300.

Winde, M., 2009. Systematische Bewertung und Ertüchtigung von industriellen Regelkreisen in verfahrenstechnischen Komplexen. Ph.D. thesis. Ruhr-Universität Bochum, Fakultät für Maschinenbau.

Zhang, Z., Wu, Z., Durand, H., Albalawi, F., Christofides, P.D., 2018. On integration of feedback control and safety systems: Analyzing two chemical process applications. Chemical Engineering Research and Design 132, 616-626. 